\title{
Synthesis and Mechanistic Interrogation of Ginkgo biloba Chemical Space en route to $(-)$-Bilobalide
}

\author{
Robert M. Demoret, ${ }^{1,+}$ Meghan A. Baker, ${ }^{1, \dagger}$ Masaki Ohtawa, ${ }^{1, \dagger}$ Shuming Chen, ${ }^{2}$ Ching Ching Lam, ${ }^{2}$ Stefano For- \\ li, ${ }^{3}$ Kendall N. Houk, ${ }^{2}$ Ryan A. Shenvi ${ }^{*}$ \\ ${ }^{1}$ Department of Chemistry, Scripps Research, 10550 North Torrey Pines Road, La Jolla, California 92037, United States \\ ${ }^{2}$ Department of Chemistry and Biochemistry, University of California, Los Angeles, CA, USA \\ ${ }^{3}$ DISCoBio, Scripps Research, 10550 North Torrey Pines Road, La Jolla, California 92037, United States
}

\begin{abstract}
Here we interrogate the structurally dense ( $\left.1.63 \mathrm{mcbits} / \AA^{3}\right) \mathrm{GABA}_{\mathrm{A}}$ receptor antagonist bilobalide, intermediates en route to its synthesis and related mechanistic questions. ${ }^{13} \mathrm{C}$ isotope labeling identified an unexpected bromine migration en route to an $\alpha$-selective, catalytic asymmetric Reformatsky reaction, ruling out an asymmetric allylation pathway. Experiment and computation converge on the driving forces behind two surprising observations. First, an oxetane acetal is shown to persist in concentrated mineral acid (1.5 M DCl in THF- $\left.\mathrm{d}_{8} / \mathrm{D}_{2} \mathrm{O}\right)$, and its longevity is correlated to destabilizing steric clashes between substituents. Second, a regioselective oxidation of des-hydroxybilobalide is found to rely on lactone acidification through lone-pair delocalization, which leads to extremely rapid intermolecular enolate equilibration. In addition, we describe multiple pitfalls, puzzles and unexpected reactions that ultimately uncovered a concise total synthesis. These problems arose from the high information density of bilobalide that distinguishes it from other scaffolds and may characterize natural product (NP) space more generally. Therefore, we also include a Python script to quickly ( $c a .132,000$ molecules/ minute) calculate information content (Böttcher scores), which may be helpful to identify important features of NP space.
\end{abstract}

Introduction. Herbal supplements and remedies constitute a large and controversial market. ${ }^{1}$ Not regulated as conventional foods or drugs by the FDA, ${ }^{2}$ plant extracts can nevertheless contain orally bioavailable small molecules with profound effects on human biology. Successful sales, however, do not rely on the same demonstrations of efficacy and safety as approved pharmaceuticals. For instance, leaf extracts of Ginkgo biloba are widely marketed today as nootropic supplements, but the ability of these extracts to improve cognition is questionable. ${ }^{3}$ Despite the ambiguity, approximately 1.6 million adults reported using G. biloba extracts in the US in a 2012 survey. ${ }^{4,5}$ Even with clear target annotation for several constituents, as well as proven brain penetration, ${ }^{6}$ any benefits of Ginkgo extracts remain largely unsubstantiated. Additionally, a two-year study by the National Toxicology Program (NTP) concluded that one commercially available G. Biloba extract increased rates of liver and thyroid cancers in mice and rats. ${ }^{7}$ Adverse events have been long attributed to ingestion of Gingko extracts, especially increased bleed time (see below).

Even so, individual components of the crude extract may find useful application if isolated from the off-target activity of congeners. For example, pure bilobalide (BB) exhibited normalization of cognitive impairment in models of Down syndrome, Ts65Dn mice, which exhibit excess inhibitory signaling. ${ }^{8}$ Four-week administration of bilobalide led to improved cognitive performance, which correlated to enhanced long-term potentiation (LTP, strengthening of synapses) by a related compound (pentyltetrazole, PTZ) even months after drug regimen had ended. Bilobalide also displayed protection against NMDA-induced excitotoxicity in rat hippocampus. ${ }^{9}$

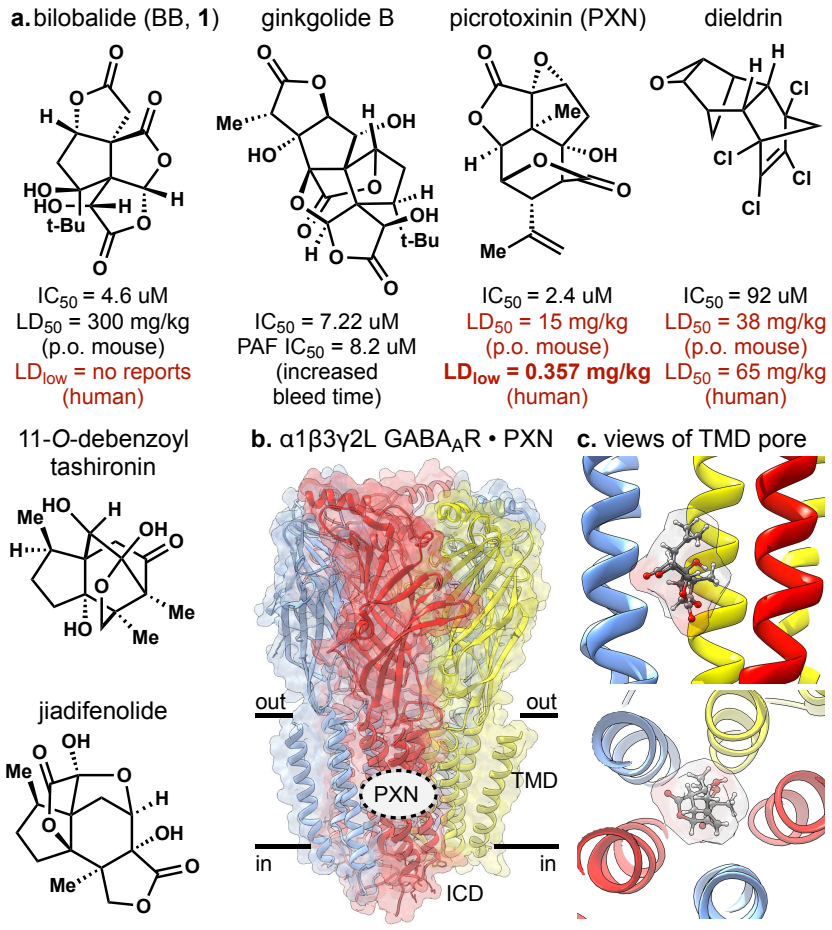

Figure 1. a. Hyperexcitatory compounds that antagonize $\mathrm{GABA}_{\mathrm{A}}$ receptors; b. cryogenic electron microscopy structure of the human $\alpha 1 \beta 3 \gamma 2 \mathrm{~L} \mathrm{GABA}_{\mathrm{A}}$ receptors; c. channel binding site of picrotoxinin, which exhibits allosteric coupling to the extracellular region (b/c, Ref. 10). 
In addition to human applications, ${ }^{11}$ ginkgolides and bilobalide exhibit insect deterrent or insecticidal activity against the fall webworm (H. Cunea, $0.31-1.25 \mathrm{mg} / \mathrm{mL}),{ }^{12 \mathrm{a}}$ codling moth (C. pomonella, $0.1 \mathrm{mg} / \mathrm{mL}),{ }^{12 \mathrm{~b}}$ brown rice planthopper $(N$. lugens, $0.17-0.26 \mathrm{ng} /$ female) and others. ${ }^{12}$ Ginkgo leaves have long been used as bookmarks to protect against page-eating insects like silverfish and booklice. ${ }^{16 \mathrm{~b}}$ The insecticidal activity of Ginkgo biloba leaves, roots and stems may have provided advantage against predation to preserve the lineage of the early Paleocene species $G$. adiantoides. ${ }^{13}$ Benign effects of bilobalide in humans - in contrast to ginkgolides ${ }^{14}$ - commend it as a potentially safe insecticide. The seemingly disparate applications of bilobalide in mammals and in insects derive from activity at homologous ion channels: RDL (resistance to dieldrin) receptors in insects and $\mathrm{GABA}_{\mathrm{A}}$ (gamma-amino butyric acid, A) receptors in mammals (Figure $1 b$ ).

Bilobalide exhibits insecticidal activity through antagonism of RDL receptors: heteropentameric ion channels gated by GABA. These ionotropic receptors mediate fast synaptic transmission in insect nervous systems. As in mature mammalian systems, the current tends to be inhibitory. ${ }^{15}$ Antagonism of these inhibitory channels, therefore, causes increase in spontaneous firing and can prove lethal. As a consequence, RDL receptors are common targets of insect-control agents, such as their namesake, dieldrin, a member of the cyclodiene class. An Ala $\rightarrow$ Ser mutation within the channel ${ }^{16}$ reduces sensitivity to GABA receptor antagonists, including picrotoxinin (PXN, the more active component of the mixed substance picrotoxin, PTX), a plant metabolite of similar formula and topology to bilobalide.

Dieldrin, PXN and bilobalide also antagonize homologous human GABA-gated ion channels or $\mathrm{GABA}_{\mathrm{A}}$ receptors $\left(\mathrm{GABA}_{\mathrm{A}} \mathrm{Rs}\right)$. Point mutation of channel residues ${ }^{17}$ identified a likely binding site for bilobalide near the same location as dieldrin and $\mathrm{PXN}^{18}$ : a wide cavity between 2' and 6' residues on the M2 helix in the transmembrane domain (Figure 1c). ${ }^{19}$ $\mathrm{BB}$ and PXN also display similar potency against a major subtype of GABA receptor in the human brain $\left(\mathrm{IC}_{50}=1.2 \mu \mathrm{M}\right.$ vs. $\left.4.9 \mu \mathrm{M}, \alpha_{1} \beta_{2} \gamma_{2 \mathrm{~L}} @ \mathrm{EC}_{50} \mathrm{GABA}\right)$, although they respond differently to diverse positive allosteric modulators. ${ }^{20}$

Both PXN and dieldrin, however, exhibit marked neurotoxicity in mammals. The latter compound, a polychlorinated pentacycle, persists in the environment, transits along the foodchain and causes multiple disorders in humans. ${ }^{21}$ In contrast, bilobalide is well-tolerated in mammals at low to moderate doses and breaks down easily near neutral $\mathrm{pH}$ (see below). As a result, there is significant interest in the development of bilobalide as an insecticide. ${ }^{12}$ Crude or semi-purified $G$. biloba extracts unfortunately contain ginkgolides A-J, which inhibit RDL receptors but also antagonize human platelet aggregating factor (PAF) and are stable to water.

We became interested in $\mathbf{1}$ due to its similarity to the Illicium sesquiterpenes, ${ }^{22}$ which hyperexcite mammalian neurons ${ }^{22 b}$ but display potentially therapeutic phenotypes in mice, such as normalization of amphetamine-induced hyperlocomotion, an antipsychotic effect. ${ }^{23} \mathrm{We}$ also recognized the opportunity to investigate broader strategies in polyoxygenated carbocycle synthesis gleaned from work on jiadifenolide ${ }^{22 a}$ and $O$ debenzoyltashironin, ${ }^{22 \mathrm{~b}}$ namely the merger of highly oxidized but sterically congested fragments to accelerate synthesis. ${ }^{24}$
Here we describe our efforts to devise a concise synthesis of bilobalide (BB, 1), a challenge due to its two hallmark features: 1) congestion, both steric and stereogenic, and 2) polyoxygenation. Together, these features give 1 a very high information density ( 1.63 mcbits/ $\AA^{3}$, vide infra). In contrast to information content (mcbits), ${ }^{25}$ which mostly reflects heavy atom count, information density (mcbits/ $\AA^{3}$ ) reflects how that information is packaged and affects emergent properties. ${ }^{26}$ The efficient conversion of low information-density building blocks into high information-density targets is a fundamental aim of total synthesis. This exercise is particularly important in channel-binding ligands of ionotropic receptors, especially terpenoids typified by $\mathbf{1}$, where atoms must be restricted to a small volume: 1 is only $260 \AA^{3}$. In comparison, the steroidderived anxiolytic minaxolone, ${ }^{27}$ which binds the extracellular region of $\mathrm{GABA}_{\mathrm{A}}$ receptors occupies $498 \AA^{3}$ and the insecticidal macrolide ivermectin, which binds the outer transmembrane region, occupies $835 \AA^{3}$. Compared to similarly-sized natural products pleuromutilin, himbacine and artemisinin, bilobalide possesses the highest information density, primarily due to its oxygen content, stereocenters and small volume (Figure 2). Stereocenters play a major role in the information density of BB: an achiral constitutional isomer contains nearly half the information density (0.94 vs. 1.63 mcbits/ $\left.\AA^{3}\right)$.

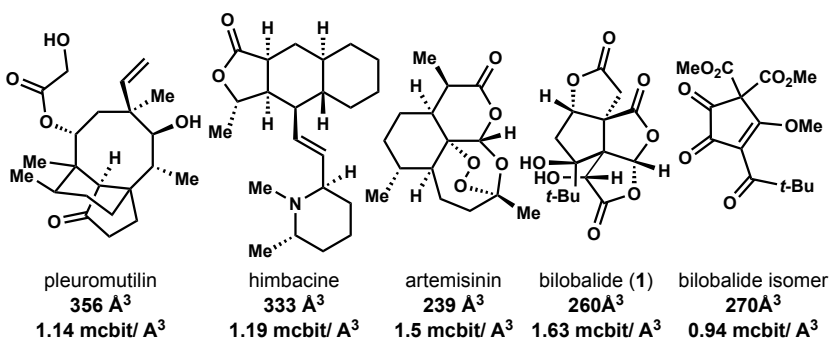

Figure 2. Representative secondary metabolites, volumes and information densities.

Two overarching strategies differentiate this work from the prior $^{28,29}$ syntheses: incorporation of oxidation state in starting materials and formation of hindered $\mathrm{C}-\mathrm{C}$ bonds between these building blocks. The result, realized retrospectively, is a generally positive increase in average information content; ${ }^{30}$ each step productively approaches the target complexity.

The execution of the synthesis relied on recursive feedback between several discoveries that we analyze here in detail. First, we provide a full account of the development of a catalytic, enantioselective $\mathrm{Zn}^{+2}$-Reformatsky aldol that overcomes the thermodynamic instability of the first key building block. Second, experimental observations and mechanistic hypotheses rationalize the development of a stereoselective Mukaiyama hydration reaction, a considerable challenge associated with selective diradical $\left(\mathrm{C} \bullet+\mathrm{O}_{2}\right)$ collapse. Third, computational analysis describes the driving forces behind the unexpected formation of an oxetane acetal. Fourth, details behind a final, deep oxidation of $\mathrm{C} 10$ are provided, including analysis of selectivity using computed $\mathrm{pKa}$.

Results and Discussion. Foundational work by $\mathrm{Corey}^{28}$ and Crimmins $^{29}$ established successful strategies to complete bilobalide and informed our own orthogonal approach. ${ }^{31}$ These prior routes established the contiguous, fully substituted centers of bilobalide early in the synthesis via open-shell intermediates. For example, Corey's synthesis installed every carbon 
atom of bilobalide in the very first step using a base-mediated annulation (Figure 3), proposed to proceed by single electron transfer followed by diradical collapse. Six of the resulting carbon atoms underwent redox manipulation during elaboration to BB. Of the 21 steps required to elaborate this intermediate to the target, 11 were redox manipulations. Similarly, Crimmins' synthesis established all four contiguous, fully substituted carbons early, at step 8 of 17, via a photochemical enone-olefin [2+2] cycloaddition. Bond formation via the enone triplet excited state $\left(\pi \rightarrow \pi^{*}\right)$, radical cyclization and subsequent 1,4-diradical ${ }^{32}$ collapse reflects some mechanistic similarity to ynoate annulation. The resulting cyclobutane contains 5 carbons at the incorrect oxidation state, requiring a total of 8 redox manipulations to reach $\mathrm{BB}$.
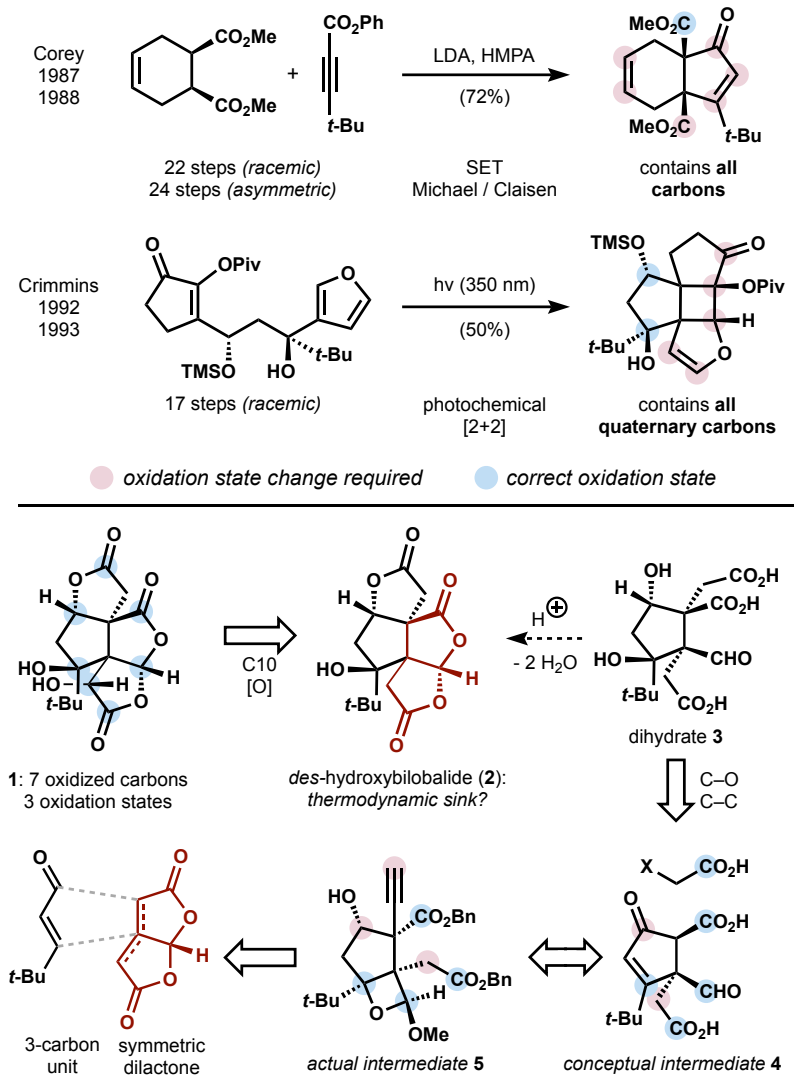

Figure 3. Previous syntheses of 1 relied on early carbon framework synthesis, followed by oxidation state changes. The work here uses mostly preoxidized building blocks.

In both cases, successful construction of the congested $\mathrm{BB}$ core can be attributed to the early transition states of radical reactions that minimize steric repulsion. A more concise synthesis might be achieved if radical reactivity were retained but oxidation patterns of BB were incorporated from the outset. The pseudosymmetry of the bilobalide bis-lactone suggested a symmetric 1,5-diester starting material that could incorporate many oxidation states and might undergo radical annulation by a 3-carbon fragment with a pendant tert-butyl group (see Figure 3 and 4). The C10 hydroxyl of BB, however, proved hard to incorporate into starting material or early intermediates (Figure 4). Therefore, our strategy also hinged on installation of the single hydroxyl late in the synthesis. This late stage oxidation, while simplifying overall, presented an inherent risk due to the topology of the $\mathrm{BB}$ ring system.

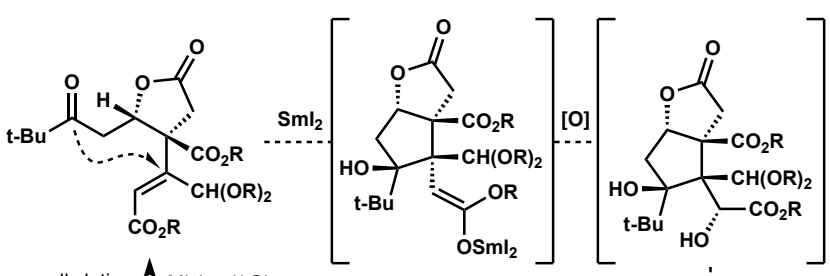

alkylation Michael/ Giese

and addition

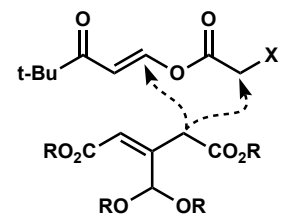

each prepared in one step

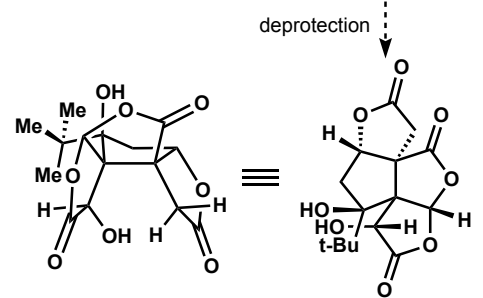

bilobalide 4 steps LLS (theoretical, not realized)
Figure 4. An early approach using a pseudosymmetric building block and radical cyclization.

The concave core of $\mathrm{BB}$ prevents easy access to either hydrogen of lactone $\mathrm{D}$ (the 'inner' lactone) in the presence of lactone A (the 'outer' lactone) (Figure 5). Instead, $\mathrm{H}_{\mathrm{k}}$ is buried deep within the 'bowl' shape of bilobalide and $\mathrm{H}_{\mathrm{i}}$ is occluded by the bulky $t$-Bu group. While retrosynthetically simplifying, this 'deep' oxidation strategy pushed a tremendous amount of risk to the endgame. If the route, however, were accomplished in the projected eight step sequence, a robust supply of deshydroxybilobalide (2) would be available for experimentation, mitigating some risk.

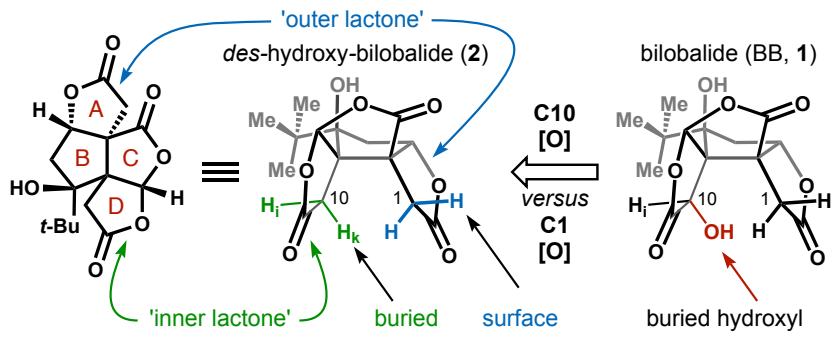

Figure 5. Structural analysis of $B B$ and identification of the topological problem with late stage oxidation.

We also hypothesized that des-hydroxybilobalide (2) might form spontaneously from its dihydrate - an aldehyde triacid - and could be a useful thermodynamic sink to target (Figure 3). ${ }^{84 a}$ Dihydrate $\mathbf{3}$ could then arise from hydration, alkylation and subsequent global condensation of cyclopentene 4 . The hindered tertiary alcohol of $\mathbf{3}$, flanked by two quaternary carbons, would be generated from the corresponding alkene using a Mukaiyama hydration. We previously proposed $^{33,34}$ that this base-metal catalyzed radical hydration proceeds via an outer-sphere, metal-hydride hydrogen atom transfer (MHAT) elementary step, and reasoned that this mechanism would tolerate the extreme steric hindrance of 4 (Figure $3)$, whereas an inner-sphere coordinative mechanism would fail. Simplified cyclopentene 4 turned out to be merely conceptual; the actual intermediate (5) ended up very different.

Initial attempts to forge the key $\mathrm{C}-\mathrm{C}$ bond between symmetric diester $\mathbf{6}$ and 7 via aldol reaction met with failure (Table 1). No product was observed upon addition of 7 to alkali enolates of $\mathbf{6}$ at cryogenic temperatures. Trace product was observed under equilibrating, basic conditions (entry 3$)^{35}$, but attempts to trap the product alcohol through alkylation, acylation or 
silylation were unsuccessful. These data indicated that the reaction equilibrium lay in favor of $\mathbf{6}$, an observation that was subsequently corroborated by the facile retro-aldol reaction of 8 under basic conditions to give 6 and 7 (entry 5).

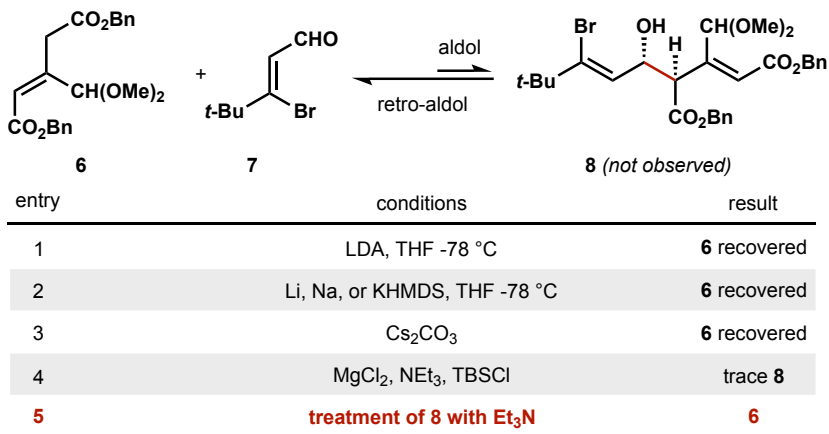

Table 1. Reversible and irreversible enolization conditions favor starting materials over product.

Instead, we anticipated that vinyl anion addition to the aldehyde would be irreversible (Figure 6) from adduct iso-8. To test this hypothesis we sought vinyl bromide iso-9. Surprisingly, Wittig reaction between bromo ylide $\mathbf{1 0}$ and $\beta$-ketoester 11 resulted in formation of allylic bromide 9, through what first seemed to be olefin isomerization (Figure 6). The symmetry of the diester, however, prevented differentiation of $\mathrm{H}$ versus $\mathrm{Br}$ migration, so a ${ }^{13} \mathrm{C}$-labelled analogue of $11\left({ }^{13} \mathbf{C}-\mathbf{1 1}\right)$ was synthesized. Following olefination, the ${ }^{13} \mathrm{C}$ labelled position bore the bromide $\left({ }^{13} \mathbf{C}-9\right)$, indicating migration of the bromide from the ylide to the $\beta$-ketoester $\left({ }^{\mathbf{1 3}} \mathbf{C}-\mathbf{1 1} \rightarrow{ }^{\mathbf{1 3}} \mathbf{C}-\mathbf{1 2}\right)$, followed by olefination. The unexpected $\alpha$-bromoester might be used in a cryogenic Reformatsky reaction that could promote stabilization of the aldol intermediate through metal chelation between a strongly-bound $\mathrm{Zn}^{+2}$ or $\mathrm{Sm}^{+3}$ alkoxide and the neighboring benzyl ester. ${ }^{36,37}$ Low temperature acidic quench could then prevent retro-aldol.
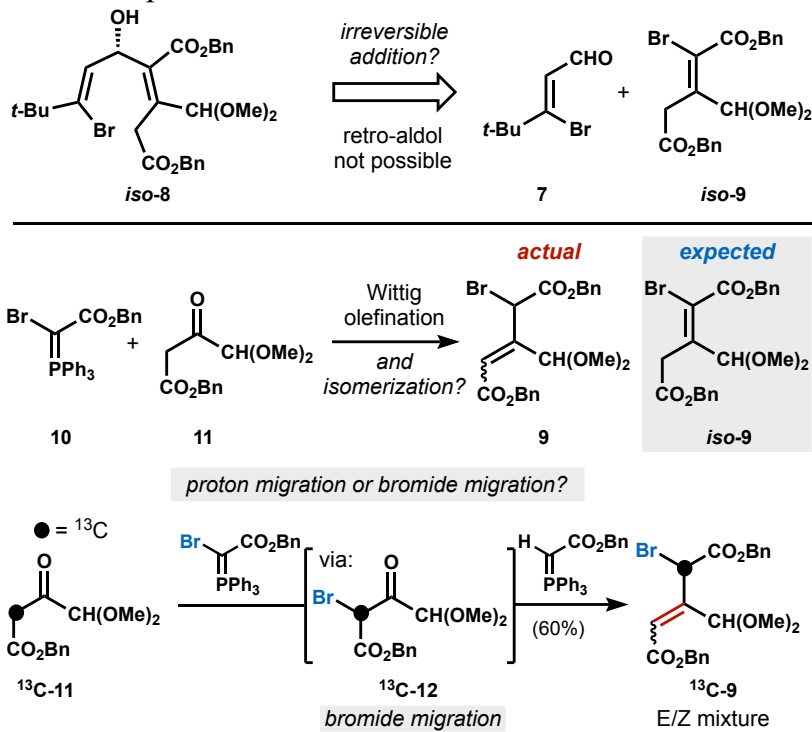

Figure 6. ${ }^{13} \mathrm{C}$ isotope labeling identifies bromide migration as operating in preference to proton migration.

Initial attempts at a Reformatsky reaction with $\mathrm{Zn}^{0}$ or $\mathrm{In}^{0}$ led only to the recovery of starting material or protodehalogenation. After extensive screening, $\mathrm{SmI}_{2}$ proved to be the ideal reductant, ${ }^{36,37}$ affording aldol product $\mathbf{8}$ in a $15: 1$ ratio over diastereomer 13 (Figure 7, entry 1 and top HPLC trace).
The reaction was reliably scaled to $51 \mathrm{mmol}$ in a single pass using $1 \mathrm{~L}$ of a $0.1 \mathrm{M}$ THF solution of $\mathrm{SmI}_{2}$ (MilliporeSigma). This $\mathrm{SmI}_{2}$-mediated Reformatsky reaction was utilized for early scale-up campaigns and route scouting, however an asymmetric synthesis of (-)-BB necessitated the development of an enantioselective Reformatsky reaction.

Early trials focused on induction of asymmetry through the use of chiral ligands with $\mathrm{SmI}_{2}$. Although the work of Proctor $^{38}$ led us to test chiral diamines, amino alcohols and cinchona alkaloids, addition of these ligands shut down $\mathrm{SmI}_{2}$ reactivity or led to unproductive protodehalogenation of $\mathbf{9}$. Chiral auxiliaries were unsuccessful due to poor stability of intermediates.

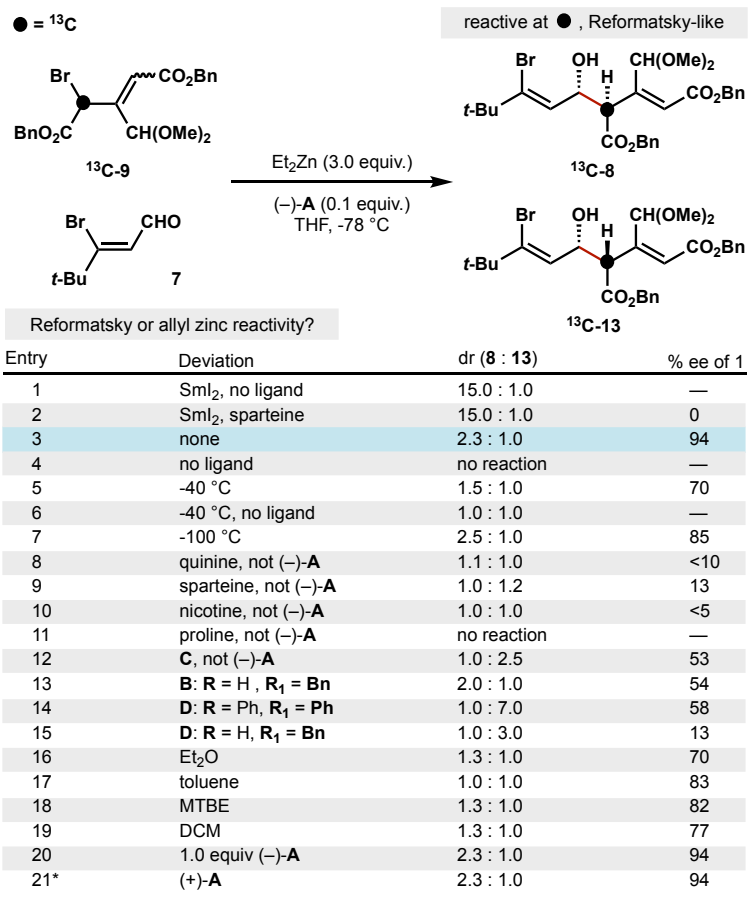

* opposite enantiomer of ligand gives opposite enantiomer of products Ligands:
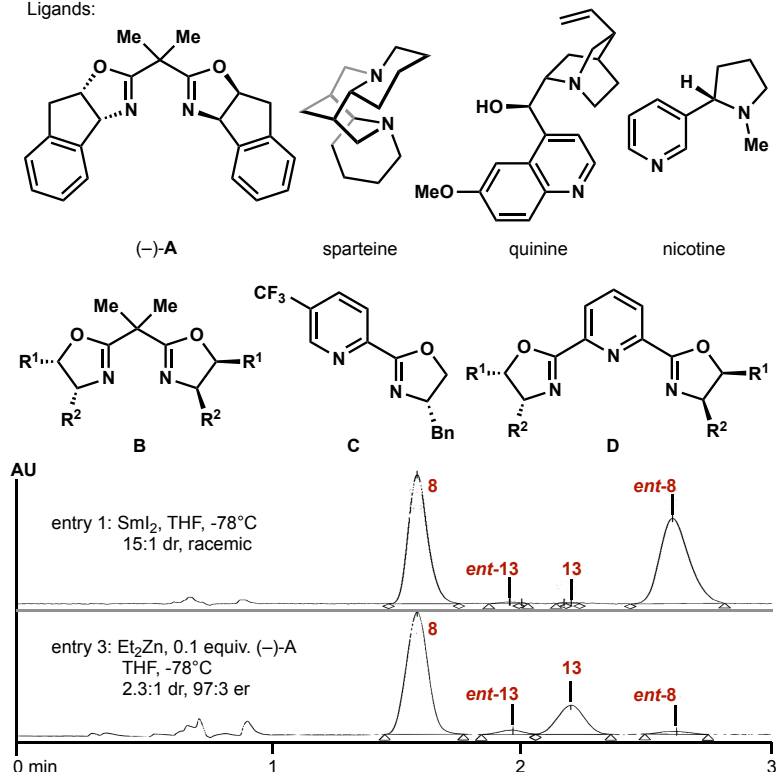

Figure 7. Development of a catalytic asymmetric Reformatsky reaction. ${ }^{13} \mathrm{C}$ isotope labeling identifies alpha-attack operating in preference to gamma-attack. 
Use of alternative metals to effect the Reformatsky reaction initially fell flat; $\mathrm{Zn}^{0}$ and $\mathrm{In}^{0}$ failed to generate product, whereas $\mathrm{Cr}^{+3}$ or $\mathrm{Cr}^{+2} / \mathrm{Ni}^{+2}$ salts led to messy reactions that were poorly diastereoselective. Diethylzinc $\left(\mathrm{Et}_{2} \mathrm{Zn}\right)$, however, resulted in clean reactions when run at $-40{ }^{\circ} \mathrm{C}$, yielding an equimolar mixture of syn- and anti-aldol diastereomers, $\mathbf{8}$ and 13 (entry 5). The undesired anti diastereomer could not be carried forward to $\mathrm{BB}$ due to formation of unproductive diastereomers in subsequent steps. Precedent for the successful use of chiral ligands ${ }^{39,40}$ with $\mathrm{Zn}^{0}$ and $\mathrm{Zn}^{+2}$ provided a foundation $^{41}$ for experimentation ${ }^{42,43,44}$, however an extensive literature search indicated a paucity of cases in which Reformatsky reactions with aldehydes are rendered both enantio- and diastereoselective ${ }^{45}$ using $\mathrm{Et}_{2} \mathrm{Zn}$. ${ }^{46}$ Most relevant methods involved primary $\alpha$-haloesters ${ }^{47,48}$, resulting in the formation of single stereocenters. ${ }^{49,50}$ This methodological gap provided an opportunity to discover new reactivity.

To this end, a number of easily accessible chiral ligands were screened in combination with $\mathrm{Et}_{2} \mathrm{Zn}$ in search of a reaction manifold that would allow for construction of two stereocenters in a single step, and in an enantio- and diastereoselective manner. Optimization data is provided in Figure 7. Unfortunately, many X-type ligands that are commonly employed in $\mathrm{Et}_{2} \mathrm{Zn}$-mediated Reformatsky reactions were unsuccessful in our system, giving either protodehalogenated starting material or complex product mixtures. The first positive results came from the use of L-type ligands including nicotine, and sparteine, and some cinchona alkaloids which generated product, albeit in low dr and ee (entries 8-10). Fortunately, combination of $\mathrm{Et}_{2} \mathrm{Zn}$ with $\mathrm{BOX}$ or PyBOX ligands led to product cleanly and in high conversion. Each ligand resulted in varying diastereomeric ratios, but often in moderate to excellent enantioselectivities. Notably, temperature significantly influenced background, racemic reaction rate: at $-78^{\circ} \mathrm{C}$ in the absence of ligand, reaction mediated by $\mathrm{Et}_{2} \mathrm{Zn}$ alone was sluggish. The racemic reaction did not compete with the ligand-catalyzed pathway until temperatures reached $-40{ }^{\circ} \mathrm{C}$. Use of indabox ligand (-)-A at $-78{ }^{\circ} \mathrm{C}$ gave the best results with a dr of 2.3:1 in favor of 8 , and an excellent $e e$ of $94 \%$ in favor of the enantiomer corresponding to nat-1. Other ligands favored the undesired diastereomer 13 but with comparable enantioselectivity. The reaction utilizing indabox ligand (-)-A could be rendered catalytic $(10 \mathrm{~mol} \%)$ and was scalable to $51 \mathrm{mmol}$ with careful control of temperature.

From the pseudosymmetric enolate of 9, two modes of reactivity could feasibly produce alcohols 8 and 13: 1) formation of an allyl zinc reagent followed by aldehyde addition via allyl-metal reactivity; or 2) via the anticipated Reformatsky-type zinc-enolate addition. ${ }^{13} \mathrm{C}$-labelled bromide ${ }^{13} \mathbf{C}-9$ differentiated these pathways in its reaction with diethylzinc and aldehyde 7 , leading to formation of secondary alcohol ${ }^{13} \mathbf{C}$ 8 and ${ }^{13}$ C-13 (2.3:1.0 dr) as single regioisomers, a result of enolate - not allyl zinc — addition. Despite the low dr, successful formation of this key carbon-carbon bond provided evidence that such an enantioselective, diastereoselective Reformatsky aldol could be rendered catalytic in ligand.

Further studies are underway to understand if a vinylogous diester is necessary for high levels of $\mathrm{dr}$ and ee or if simpler secondary $\alpha$-bromo esters can be used in this general reaction manifold. To the best of our knowledge, this represents the first example of a diastereo- and enantioselective Reformatsky reaction between a zinc enolate and an aldehyde.

Access to $\mathbf{8}$ set the stage for a 5-exo-trig Giese addition via an $\mathrm{sp}^{2}$-hybridized radical, which we anticipated would kinetically outperform the lower-energy ketyl radical (see Figure 4 ). Whereas ambient temperature cyclizations proved unsuccessful, elevated temperatures employing standard conditions (1.5 equiv. $\mathrm{Bu}_{3} \mathrm{SnH}, 0.1$ equiv. AIBN, $85{ }^{\circ} \mathrm{C}$ ) afforded the cyclopentene as a single diastereomer. The reaction likely proceeds through the transition state of intermediate 15 (Figure 8) to alleviate the syn-pentane interaction (see 16) between the dimethyl acetal and the benzyl ester. Attempts to install C10 oxidation at this stage were also unsuccessful; ${ }^{51}$ instead, radical chain propagation by $\mathrm{Sn}-\mathrm{H}$ atom transfer proved efficient. As an aid to bulk preparation, the desired product could be crystallized from the crude reaction mixture with hot pentanes, obviating the need for large-scale chromatography. One representative run produced 8.1 grams of high purity $\mathbf{1 4}$ (>99\% ee according to chiral SFC analysis).
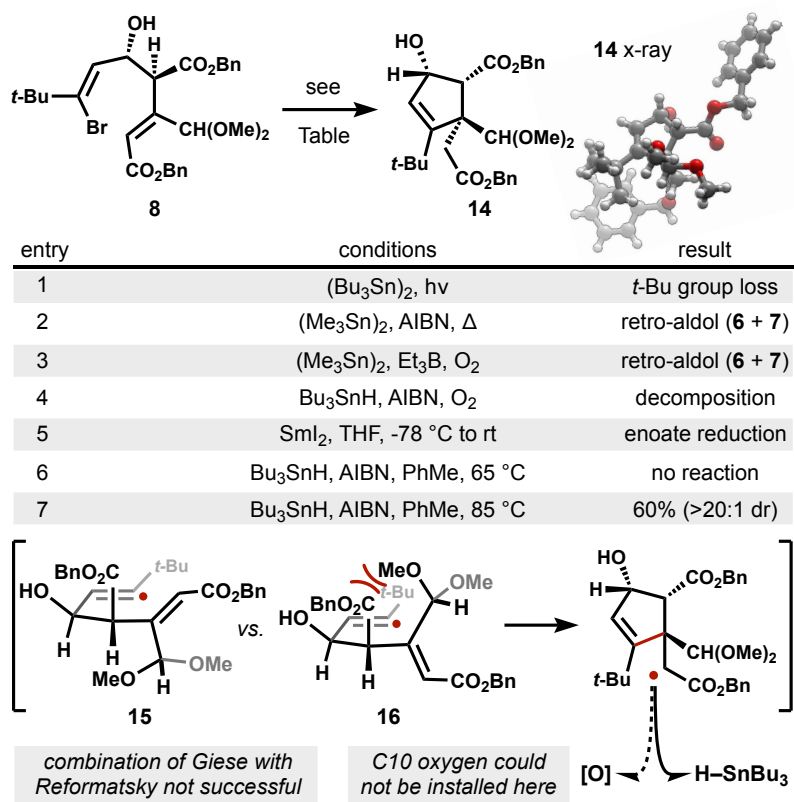

Figure 8. Diastereoselective Giese addition.

Formation of the triad of adjacent fully substituted carbon atoms at C3, C8 and C9 entailed Markovnikov hydration of 14 (Figure 9). In a prior synthesis of $\mathrm{BB}$, this transformation proved challenging and required a multi-step sequence including olefin dihydroxylation $\left(\mathrm{OsO}_{4}\right.$, pyridine) and selective mono-deoxygenation (1. methyl oxalyl chloride, $i$ - $\operatorname{Pr}_{2} \mathrm{NEt} ; 2$. AIBN, $\left.n-\mathrm{Bu}_{3} \mathrm{SnH}\right)$. Notably, osmium tetroxide dihydroxylation tolerates extreme steric hindrance ${ }^{52}$ due to its 'outersphere' $[3+2]$ cycloaddition mechanism, ${ }^{53}$ whereas coordinative 'inner-sphere' reactions of hindered alkenes can suffer very slow rates. ${ }^{54}$ Inspired by the steric tolerance of outersphere elementary steps, we explored hydration under DragoMukaiyama conditions. ${ }^{34}$ These base-metal catalyzed reactions have garnered significant attention due to their 'mild' conditions, Markovnikov selectivity and ability to tolerate sterically demanding systems. Their extensive use across unique, challenging chemotypes embodies what we call high "chemofidelity': reliable reactivity, independent of molecular context. ${ }^{55}$ Success here relies on the 'outer-sphere' nature of the MHAT 
mechanism, since the most hindered carbon center does not require a bonding interaction in the transition state, but instead is converted directly to a carbon centered radical.

Unfortunately, the use of standard Mukaiyama hydration conditions produced a near equal mixture of diastereomers and unexpected byproduct 19, which lacked the tert-butyl group (Figure 9). The low diastereoselectivity reflected capture of the open-shell carbon with ${ }^{3} \mathrm{O}_{2}$, and the byproduct resulted from either Wieland (radical) or Criegee (cationic) fragmentation. ${ }^{56}$ Either way, we expected that a decrease in the concentration of intermediate peroxide would minimize 19. A seminal mechanistic study by Nishinaga et al. on the mechanism of Drago's cobalt salen-catalyzed hydration reported rate acceleration by the addition of triphenylphosphine (10-fold relative to cobalt), which was interpreted to result from the reduction of an intermediate cobalt-peroxide. ${ }^{57}$ The hydration of 14 catalyzed by $\mathrm{Co}(\mathrm{acac})_{2}$, however, did not benefit from the addition of $\mathrm{PPh}_{3}$. Instead, no hydration occurred until all $\mathrm{PPh}_{3}$ had been oxidized to $\mathrm{O}=\mathrm{PPh}_{3}$, at which point the same poor reaction mixture resulted! As a result, a series of cobalt, manganese and iron catalysts - all precedented by Mukaiyama to catalyze

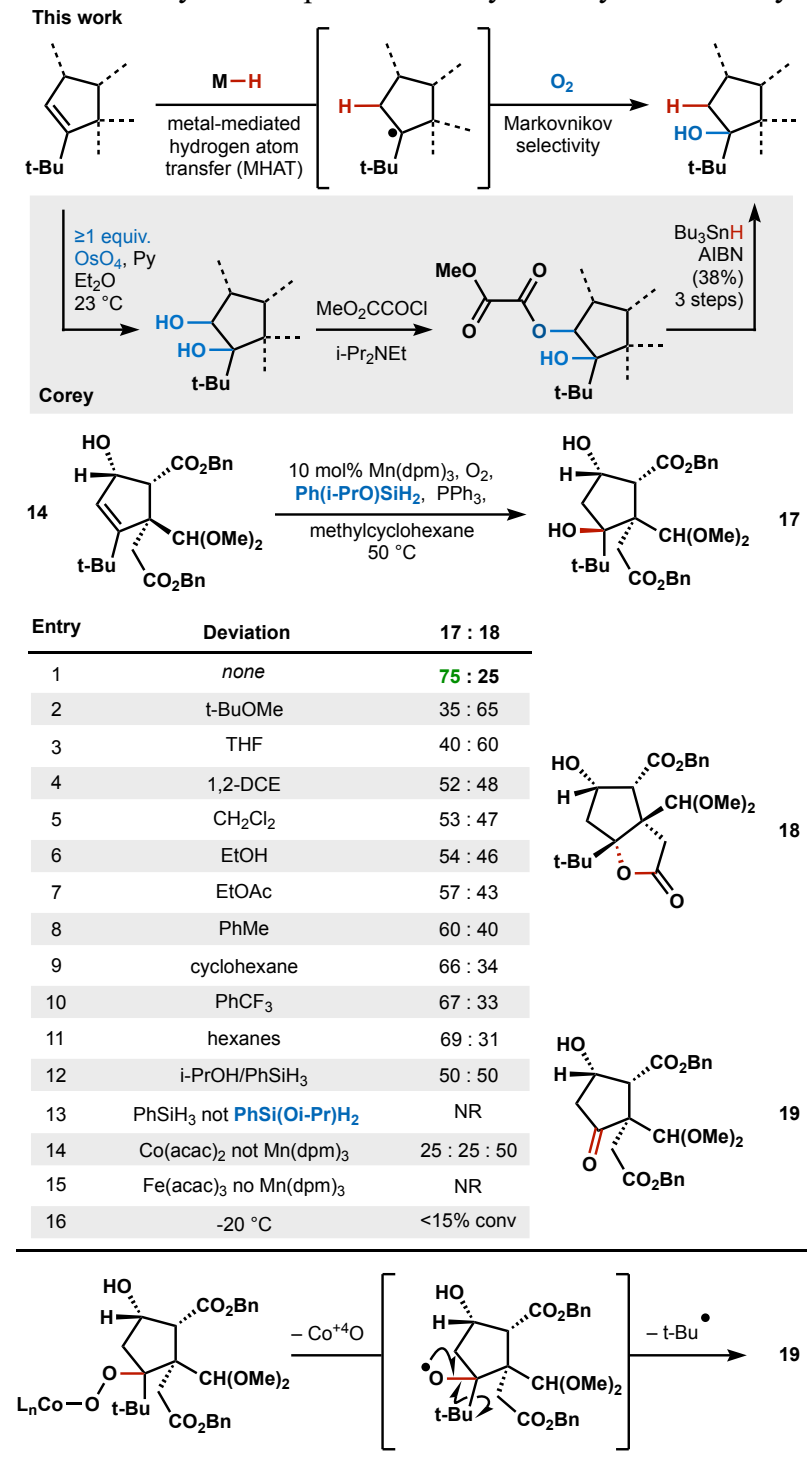

Figure 9. Effect of solvent, metal center and phosphine on Mukaiyama hydration probed using $\mathrm{Ph}(i-\mathrm{PrO}) \mathrm{SiH}_{2}$. the hydrofunctionalization of alkenes - were screened for performance in the presence of $\mathrm{PPh}_{3} . \mathrm{Mn}(\mathrm{dpm})_{3}$ proved optimal but sensitive: $\mathrm{PPh}_{3}$ allowed catalysis and completely suppressed the formation of 19 , yet its omission led to 19 exclusively. The equivalent reaction rate $\mathbf{1 4}$ in the presence of absence of $\mathrm{PPh}_{3}$ suggested that it did not react with any kinetically relevant species in the Mn-catalyzed reaction. In contrast, $\mathrm{PPh}_{3}$ shut down the productive reaction pathway of cobalt. Additionally, a variety of $\mathrm{Mn}, \mathrm{Fe}$ and Co $\beta$-diketonate complexes showed no variation in diastereoselectivity, reflecting the outer-sphere MHAT mechanism and capture of dioxygen by a carbon radical.

This discouraging selectivity exposed the inherent limitation of MHAT reactions: substrate dependent stereoselectivity in almost all cases. ${ }^{58}$ Solvent screens were possible using cobalt catalysis, ${ }^{59}$ but $\mathrm{Mn}$ and $\mathrm{Fe}$ catalysts typically required an alcoholic solvent to function. Our lab found that these catalysts required alcohol because the standard reductant, $\mathrm{PhSiH}_{3}$, performed poorly compared to its mono-alcoholysis product, isopropoxy(phenyl)silane, a highly efficient reductant that functions in numerous solvents. ${ }^{60}$ This reductant allowed an extensive solvent and temperature screen, which to date, had not been reported. It was not clear, however, that solvent choice would increase the stereoselectivity of carbon radical capture by $\mathrm{O}_{2}$.

To our surprise, solvent played a significant role in stereoselectivity and correlated roughly to Lewis basicity. Ethereal solvent favored the undesired diastereomer 18, which lactonized due to proximity of the southern benzyl ester. As solvent polarity decreased, the reaction increasingly favored diastereomer 17. Methylcyclohexane proved to be the optimal solvent and afforded $\mathbf{1 7}$ as the major diastereomer, isolable in $67 \%$ yield. These data contrast a recent paper from the Woerpel group that demonstrated small effects of solvent on $\mathrm{dr}$ $(85: 15 \rightarrow 97: 3)$, the benefits of which were mitigated by decreases in yield $(60 \rightarrow 29 \%)$. In this case, stereoselectivity was explained by a chair versus twist boat, Fürst-Plattner-like model and required a six-membered ring (Figure 10). Solventbased stereochemical reversal of five-membered ring hydration $(\mathbf{1 4} \rightarrow \mathbf{1 7})$, however, is hard to explain with this model. Instead, stereoselectivity might be explained by an internal hydrogen bond between the C6 secondary alcohol and the C5 ester (Figure 10) that is strengthened in conformation 20. Nonpolar solvents like methylcyclohexane could increasingly favor this interaction, whereas hydrogen bond acceptors like ethers could disrupt it. ${ }^{61}$

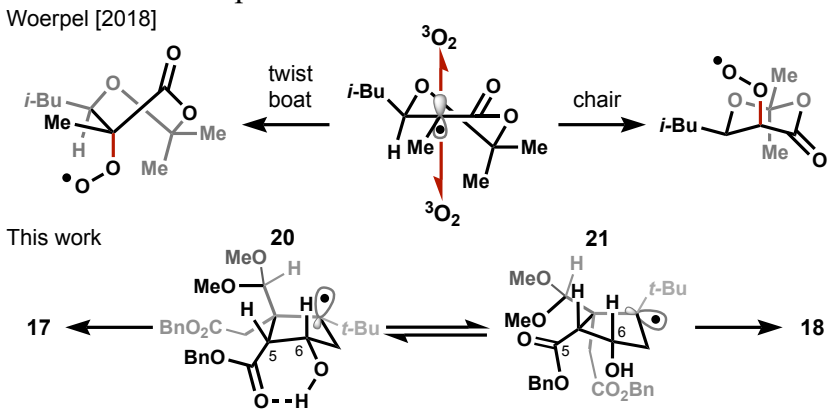

Figure 10. Stereoselectivity via proposed chair versus twistboat transition state conformations, compared with interconverting envelopes. 
These effects highlight the inherent substrate dependent diastereoselectivity in the Mukaiyama hydration, and emphasize how solvent effects can perturb the conformational equilibria of pyramidalized radicals. This represents the first example of a solvent dependence dictating the stereoselectivity of the Mukaiyama hydration, made possible by iso-propoxy(phenyl)silane.

Only a single substitution of the cyclopentane now remained to reach subtarget 3 (Figure 3): stereoselective connection to an acetic acid unit at C5. Although acylation of the C6 secondary alcohol was achieved with EDCI/ bromoacetic acid, cyclization of $\mathbf{2 2}$ to $\mathbf{2 4}$ under basic conditions was completely outcompeted by E1cB elimination to 23. Attempts to choreograph lactone formation earlier, prior to Mukaiyama hydration, also proved unsuccessful and $\mathbf{2 5}$ yielded elimination product 26, instead of 27. A number of attempts were made at direct alkylation of $\mathbf{1 4}$ by a two-carbon unit via Frater-Seebach alkylation ${ }^{62,63}$ with methyl bromoacetate. However, treatment of the alkoxy ester 14 with 2 equivalents of strong base still led to elimination, leaving the question of stereoselectivity open-ended.
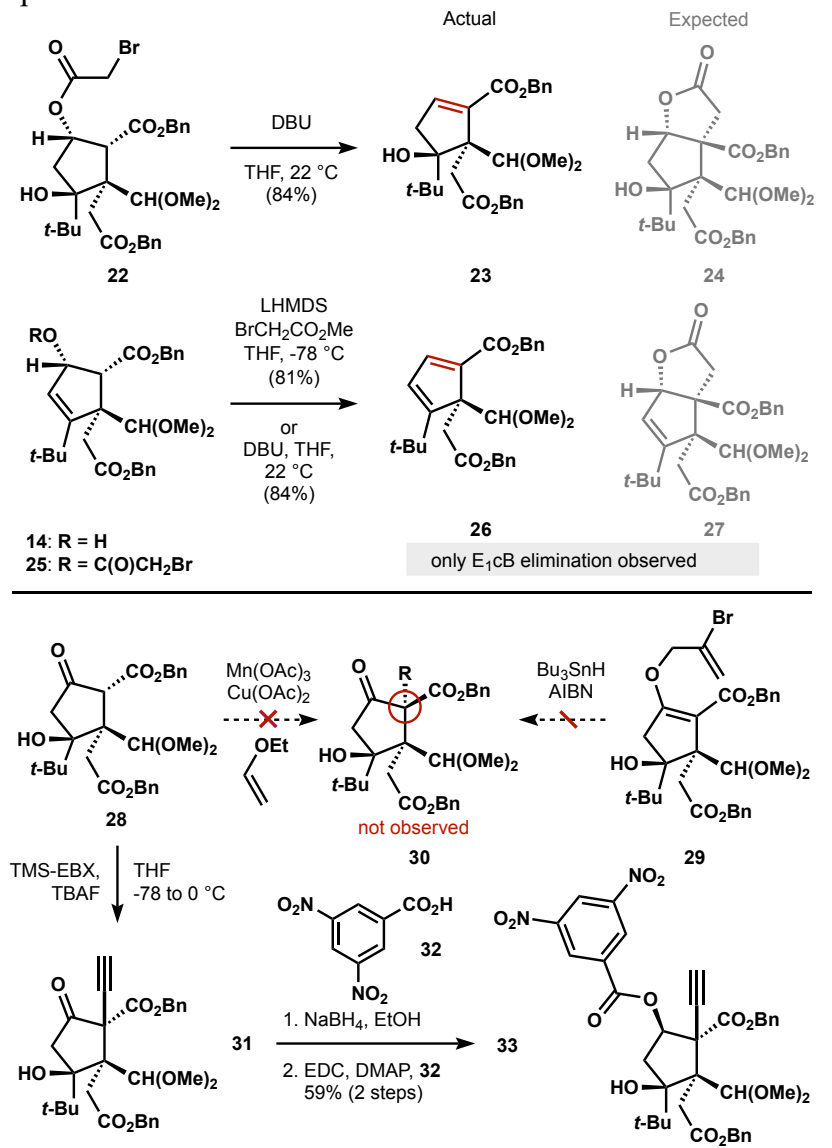

Figure 11. Attempts to install final two-carbon unit at $\mathrm{C} 5$.

To prevent $\mathrm{E} 1 \mathrm{cB}$ elimination and probe substrate control over the stereoselectivity of $\mathrm{C}-\mathrm{C}$ bond formation, alcohol $\mathbf{1 4}$ was oxidized to $\beta$-ketoester 28. A variety of electrophiles proved incapable of reacting with the extremely hindered carbon $\mathrm{C} 5$. $\mathrm{O}$-acylation occurred to deliver enol bromoacetate $\mathbf{2 9}$, but radical cyclization to $\mathbf{3 0}$ did not. As a result of the congested steric environment, we explored sp-hybridized electrophiles, including Waser's reagent (TMS-EBX). ${ }^{64}$ This isolable alkyne electrophile desilylates with TBAF to deliver the high- ly reactive parent compound ethynyl benziodoxolone (EBX), which has been isolated recently as its acetonitrile complex. ${ }^{65}$ Together, its electrophilicity and small size allowed bond formation at $\mathrm{C} 5$ where many other reagents failed. To our frustration, however, the $R$-isomer $\mathbf{3 1}$ predominated from cyclopentanone $28{ }^{66}$

To reverse this stereoselectivity, we pursued formation of the BB bis-lactone earlier than planned $(\mathbf{1 4} \rightarrow \mathbf{3 4} \rightarrow \mathbf{3 5}$, Figure 12), which we thought might direct the delivery of EBX to the correct re-face, at that point a convex surface, of the BB partial ring system. To our surprise, attempted global deprotection of the bis-ester acetal 14 with para-toluenesulfonic acid delivered an extremely unreactive oxetane acetal as a 1:3 mixture of diastereomers 36 and 37, both resistant to epimerization.
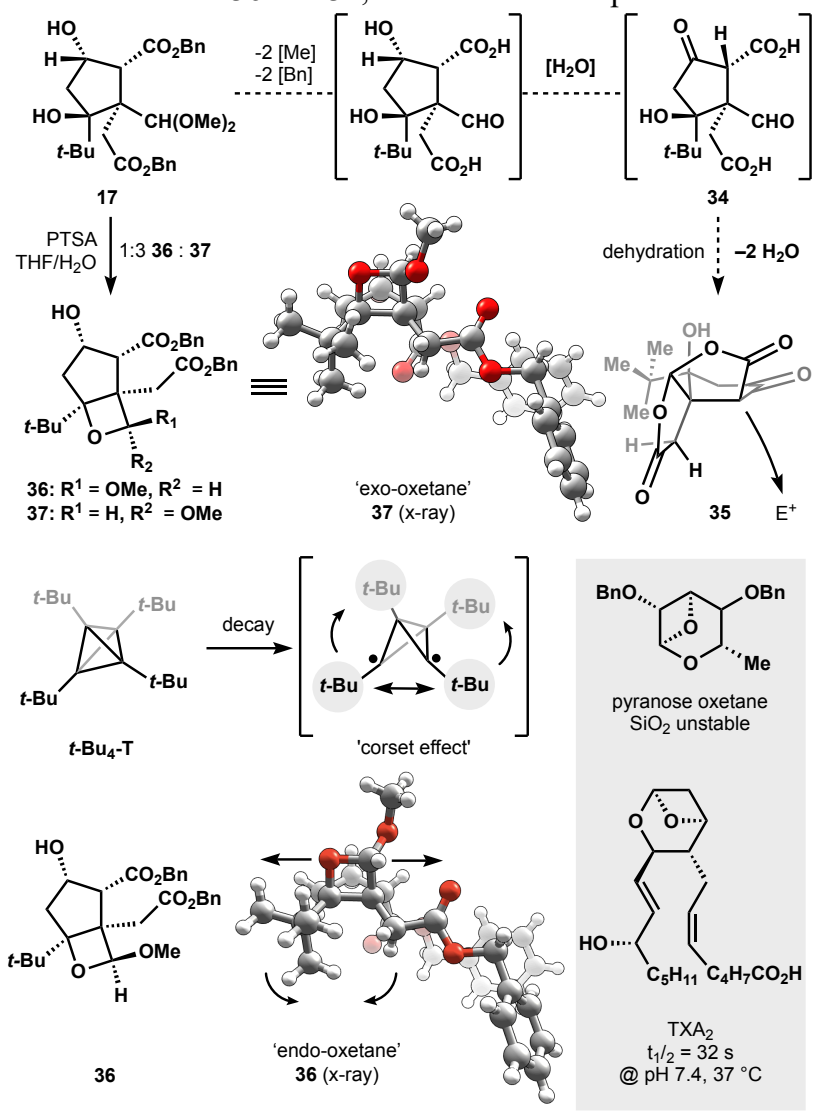

Figure 12. Unexpected trans-acetalization to an acid-stable oxetane acetal driven by Maier's hypothetical 'corset' effect, where bulky groups destabilize the bond-lengthening decomposition.

The oxetane acetal motif is rare and represented by only a handful of examples in the literature. For example, the human derived platelet aggregation factor thromboxane A2 $\left(\mathrm{TXA}_{2}\right)^{67}$ contains a cyclic oxetane acetal, but degrades with a half-life of $32 \mathrm{~s}$ at $37{ }^{\circ} \mathrm{C}$ in $\mathrm{pH} 7.4 \mathrm{Krebs}$ media. Its instability complicates isolation and requires storage in basic methanol or as the alkali metal salt; the free acid decomposes within hours via oxetane ring opening. This instability is not surprising given the strain energy of oxetanes $\left(E_{\text {strain }} \sim 25 \mathrm{kcal} / \mathrm{mol}\right),{ }^{68}$ similar to epoxides (27), ${ }^{68}$ cyclobutanes (26) and cyclopropanes (28), but substantially less stable than tetrahydrofurans $(6 \mathrm{kcal} / \mathrm{mol})$. The oxetane acetals of both $\mathrm{TXA}_{2}$ and dehydrated rhamnopyranoses ${ }^{69}$ are formed under basic conditions as a result of acid lability. In addition to $\mathrm{TXA}_{2}$, two other naturally-occurring 
oxetane acetals have been isolated: oxetanocin A (a hemiaminal $)^{70}$ and maoecrystal $\mathrm{I}^{71}$ however neither compound appears to possess a facile path to decomposition via oxetane ring-opening.

In contrast, oxetanes 36 and 37, we thought, could easily open via formation of a methyl oxocarbenium ion, which could then either hydrolyze or demethylate. Its stability, however, might reflect a general feature of the bilobalide scaffold. A similar, albeit internal, acetal was reported recently as an intermediate in an approach towards the synthesis of bilobalide and survived a strongly acidic, oxidizing step $\left(\mathrm{CrO}_{3}\right.$, $\mathrm{H}_{2} \mathrm{SO}_{4}$, acetone). ${ }^{72}$ Our attempts to deprotect or epimerize the oxetane acetals $\mathbf{3 6}$ and $\mathbf{3 7}$ led to recovery of starting material, and caused us to investigate their unusual persistence.

Steric congestion imparted by the multiple quaternary carbons and tert-butyl substituent could be considered the defining idiosyncrasy of the bilobalide ring system, and one which might reasonably play a role in oxetane persistence. Such an effect is well precedented in the synthesis and study of tetrahedranes, including tetra-tert-butyl tetrahedrane $\left(\boldsymbol{t}-\mathbf{B} \mathbf{u}_{4} \mathbf{T}\right)$, which despite its strain energy $\left(E_{\text {strain }} \sim 130 \mathrm{kcal} / \mathrm{mol}\right)$ melts at $135{ }^{\circ} \mathrm{C} .{ }^{73}$ This requirement of steric congestion was attributed to the 'corset effect' whereby bond scission increases repulsive steric interaction in the transition state, leading to a high energy barrier. It was not clear whether the unusual stability of 36 and 37, however, derived from substrate stabilization, transition state destabilization or product destabilization. $\begin{aligned} & 36_{\text {calc }}: R^{1}=\mathrm{OMe}, \mathrm{R}^{2}=\mathrm{H} \\ & 37_{\text {calc }}: R^{1}=\mathrm{H}, \mathrm{R}^{2}=\mathrm{OMe}\end{aligned}$
$39 \mathrm{a}-\mathrm{d}$

\begin{tabular}{lcccccc}
\hline & \multicolumn{2}{c}{ DMSO } & \multicolumn{2}{c}{ THF } & \multicolumn{2}{c}{$\mathrm{H}_{2} \mathrm{O}$} \\
\multicolumn{1}{r}{$\mathrm{R}^{3}$} & $\mathbf{3 6}_{\text {calc }}$ & $\mathbf{3 7}_{\text {calc }}$ & $\mathbf{3 6}_{\text {calc }}$ & $\mathbf{3 7}_{\text {calc }}$ & $\mathbf{3 6}_{\text {calc }}$ & $\mathbf{3 7}_{\text {calc }}$ \\
\hline a: $\boldsymbol{t}$-Bu & +2.2 & +2.1 & +2.0 & +2.1 & +5.6 & +5.2 \\
b: $\boldsymbol{i}-\mathbf{P r}$ & -0.2 & -0.1 & -0.5 & -0.2 & +1.1 & +3.3 \\
c: $\mathbf{M e}$ & -2.0 & -2.4 & -2.3 & -2.4 & +1.2 & +1.0 \\
d: $\mathbf{H}$ & -9.4 & -9.1 & -9.5 & -9.0 & -5.5 & -6.1 \\
\hline
\end{tabular}

Figure 13. Calculated reaction free energies ( $\mathrm{kcal} / \mathrm{mol})$ for the hydrolysis of oxetane acetals at the $\omega$ B97X-D/6-311++G(d,p), $\mathrm{SMD} / / \omega \mathrm{B} 97 \mathrm{X}-\mathrm{D} / 6-31+\mathrm{G}(\mathrm{d}, \mathrm{p})$ level of theory.

Density functional theory (DFT) calculations probed whether the unusual stability of the oxetane acetal was kinetic or thermodynamic in origin. Whereas a significant barrier to protolytic ring-opening could not be found, hydrolysis was calculated to be thermodynamically uphill for $\mathbf{3 6}$ and $\mathbf{3 7}$ in DMSO, THF and water (Figure 13), despite the expected release of considerable ring strain. Replacement of the tert-butyl group with progressively smaller alkyl substituents revealed its key role in tipping the thermodynamic balance against the hydrolyzed free aldehyde 39a-d. With an isopropyl substituent, the proximal ester group was free to assume a conformation that avoided clash with the distal ester group. In contrast, a tertbutyl substituent in the same position forced the two ester moieties into close proximity with each other, creating repulsion that destabilized ring-opened form 39a (Figure 13).

These effects were analogous to Maier's corset effect: strain-release through small ring-opening was offset by

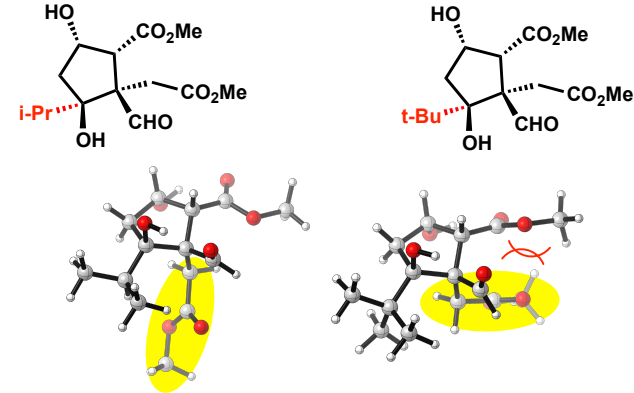

Figure 14. Calculated structures of aldehydes 39a and 39b at the $\omega \mathrm{B} 97 \mathrm{X}-\mathrm{D} / 6-31+\mathrm{G}(\mathrm{d}, \mathrm{p})$ level of theory.

destabilizing repulsion between substituents. An alternative hypothesis suggested by calculated Intrinsic Reaction Coordinates (IRCs) involved formation of stable oxocarbenium intermediates $\mathbf{4 0}$ or $\mathbf{4 1}$, which might persist in acidic media but revert to oxetane on basic work-up. This hypothesis was probed by monitoring 36 in situ. In fact, the oxetane acetals themselves were remarkably persistent, even in concentrated, strong acid. As shown in Figure 15, both $\mathbf{3 6}$ and later intermediate 48 were stored in $1.5 \mathrm{M} \mathrm{DCl}\left(1: 1 \mathrm{THF}-\mathrm{d}_{8} / \mathrm{D}_{2} \mathrm{O}\right)$ for over $24 \mathrm{~h}$ with no change in their ${ }^{1} \mathrm{H}$ NMR spectra. The lack of epimerization, oxocarbenium formation or hydrolysis indicated either a high kinetic stability or rapid internal return of a thermodynamically less-stable oxocarbenium 36 or 37 .

a. Alternative hypothesis: stable oxocarbenium and return to oxetane

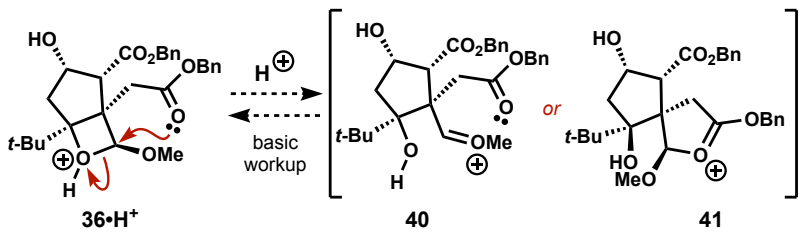

b. Pathway suggested by calculated Intrinsic Reaction Coordinates (IRCs)

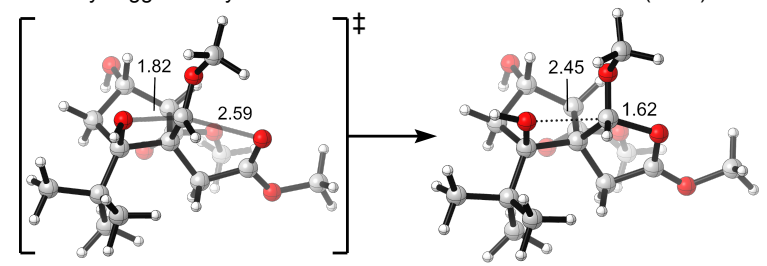

c. Exclusion of persistant oxocarbenium by in situ monitoring

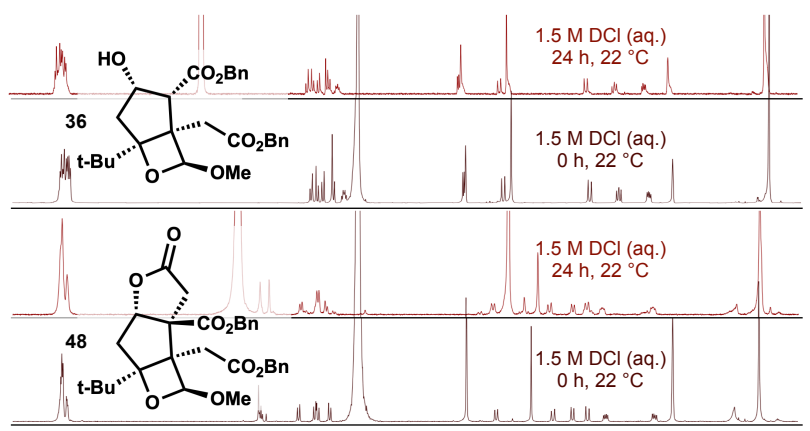

d. Conclusion: high barrier or rapid "internal return"

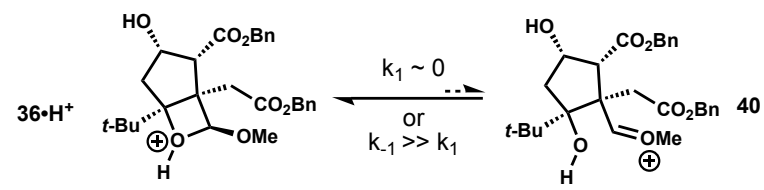

Figure 15. The alternative hypothesis of an intermediate oxocarbenium that persists in acidic media, but reverts to oxetane in basic media, was excluded. 


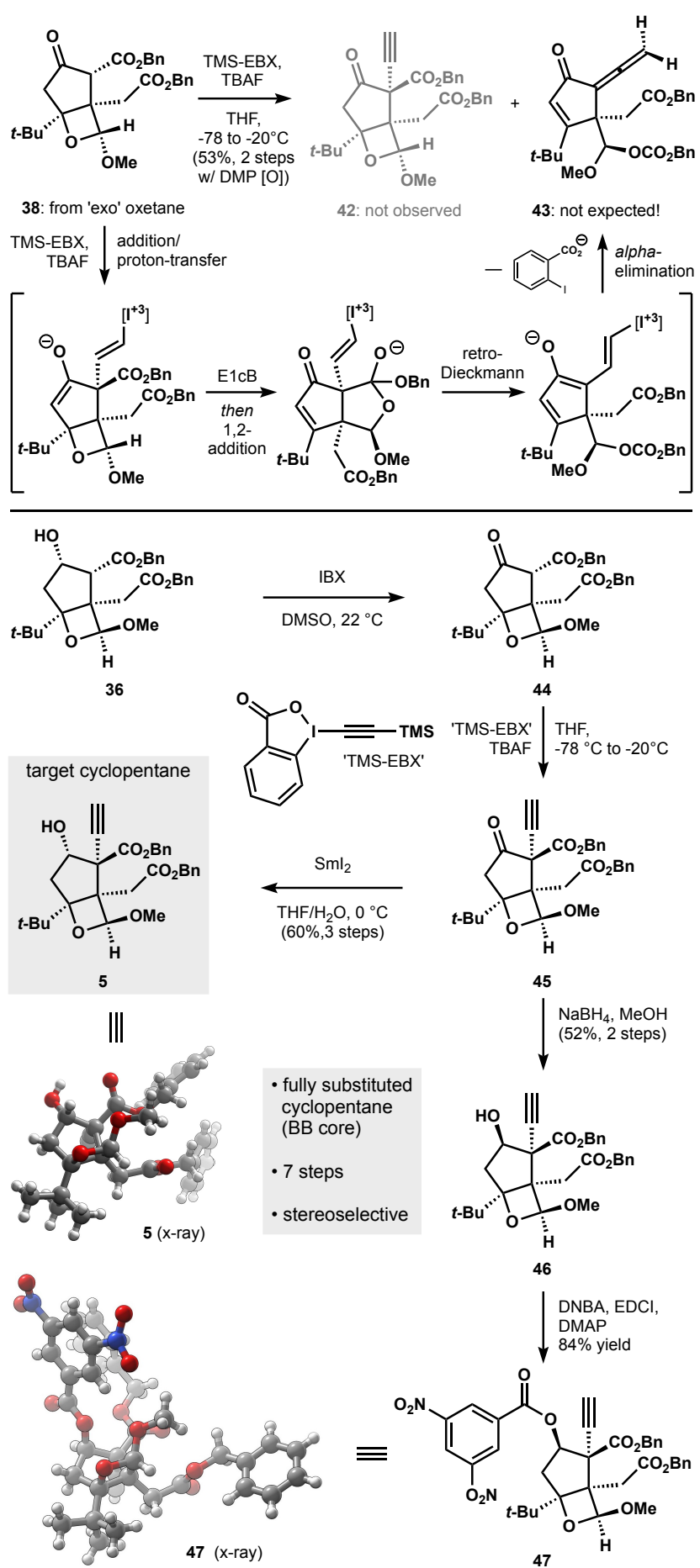

Figure 16. endo-Oxetane 36 allows stereoselective alkynylation whereas exo-oxetane 37 rearranges. Hydride reductants deliver the wrong stereoisomer but $\mathrm{SmI}_{2}$ accesses the fully substituted cyclopentane 48, a total of 7 steps from commercial materials.

The oxetane acetal motif proved more than an idiosyncrasy: its deformation of the bilobalide ring system ultimately allowed for completion of the synthesis, with one caveat. Only endo-methoxy oxetane $\mathbf{3 6}$, the minor isomer $(20 \%$ isolated, using $\mathrm{TsOH} \cdot \mathrm{H}_{2} \mathrm{O}$ ), could be advanced to bilobalide.

We had hoped that the oxetane would effectively shield the enolate $s i$-face from attack to produce alkyne $\mathbf{4 2}$. Unfortunate- ly, treatment of exo-methoxy oxetane 37 with IBX followed by Waser's reagent only afforded rearrangement product $\mathbf{4 3}$. Minor isomer 36, on the other hand, could be oxidized to $\mathbf{4 4}$ and alkynylated to $\mathbf{4 5}$ in good yield $(\geq 61 \%)$ and as a single diastereomer.

After IBX-mediated oxidation and TMS-EBX-mediated alkynylation, the synthesis required return to the alcohol oxidation state. This task was complicated by the instability of $\beta$ ketoester 45, which decomposed on silica or prolonged standing, and the tendency of the ketone to undergo reduction to $\mathbf{4 6}$ from the si-face of $\mathrm{C} 6$ by hydridic reagents, as confirmed by $\mathrm{x}$-ray crystallography $(47 \mathrm{x}$-ray). Inspired by a proposed directed reduction in Evans' synthesis of cytovaricin, ${ }^{74}$ we explored $\mathrm{SmI}_{2}$, which had been proposed to favor stereoisomers that could accommodate a chelated organosamarium(III) intermediate. In this case, chelation to the Lewis basic oxetane acetal might direct the reduction. In accordance with Evans' model, stereoisomer 5 was formed with high $(>20: 1)$ selectivity (see 5, x-ray). This key sub-target (see Figure 3) contained all the carbon-carbon bonds of $\mathbf{1}$ and required seemingly simple hydration/dehydration chemistry to complete the target, yet derived from the minor diastereomer (36) of oxetane.

Attempts to reverse the stereoselectivity of oxetane acetal formation $(\mathbf{1 7} \rightarrow \mathbf{3 6} / \mathbf{3 7})$ were initially unsuccessful. Neither solvents, simple Brønsted acids or Lewis acids led to an excess of 36. However, we observed that certain chiral phosphoric acids afforded a modest dr of $1.7: 1.0$ in favor of 36 . Given the cost of these catalysts and the low selectivity, such a solution seemed impractical. However, when working with racemic material (i.e. rac-17), we observed by chiral supercritical fluid chromatography (SFC) that each diastereomer was enriched in opposing enantiomers: chiral phosphoric acid (-)-A in a mixture of THF and $\mathrm{H}_{2} \mathrm{O}$ led to exo-oxetane $\mathbf{3 7}$ in 69:31 er and endo-oxetane $\mathbf{3 6}$ in 39:61 er. In other words, each enantiomer of $\mathrm{rac}-\mathbf{1 7}$ reacted with catalyst (-)-A to give opposing diastereomeric ratios (see Figure 17): (-)-17 favored exo-isomer $37(29: 21)$ and $(+)-17$ favored endo-isomer 36 (39:11).

These observations suggested that a parallel kinetic resolution had occurred during oxetane acetal formation. ${ }^{75}$ This effect is consistent with enantio-differentiation of prochiral acetals by chiral phosphoric acids reported by the List group. ${ }^{76}$ The same report noted that a partial parallel kinetic resolution occurred with a substrate bearing a tertiary alcohol.

In collaboration with the Scripps Automated Synthesis Facility, we developed a two-dimensional (2D) liquid chromatography (LC) / chiral SFC separation to directly probe effects of solvent, temperature and catalyst structure on the efficiency of PKR (Figure 17). Consequently, crude racemic reaction mixtures divulged their selectivity profile (dr, er) without purification. ${ }^{77}$ Pure, internal 'slices' of each $1^{\text {st }}$ dimension (LC) peak were internally directed to a chiral SFC system that separated the enantiomers of each diastereomer, quickly providing the user with ratios of all four stereoisomers of interest. A similar 2D method was developed during optimization of the Reformatsky reaction.

Multiplication of dr by er of each diastereomer derived from rac-17 delivered the ratio of endo and exo diastereomers for each enantiomer of 17. Reactions of enantiopure (+)-17 produced diastereoselectivities that corresponded well to those derived from 2D analysis and deconvolution. This method 
allowed us to work in parallel: we optimized the diastereoselectivity for $\mathbf{1 7} \rightarrow \mathbf{3 6}$ while the enantioselective Reformatsky $(\mathbf{9} \rightarrow \mathbf{8})$ was under development and $(+)-\mathbf{1 7}$ was not readily available.

a Workflow to determine dr imparted by acid on each enantiomer of a rac-17

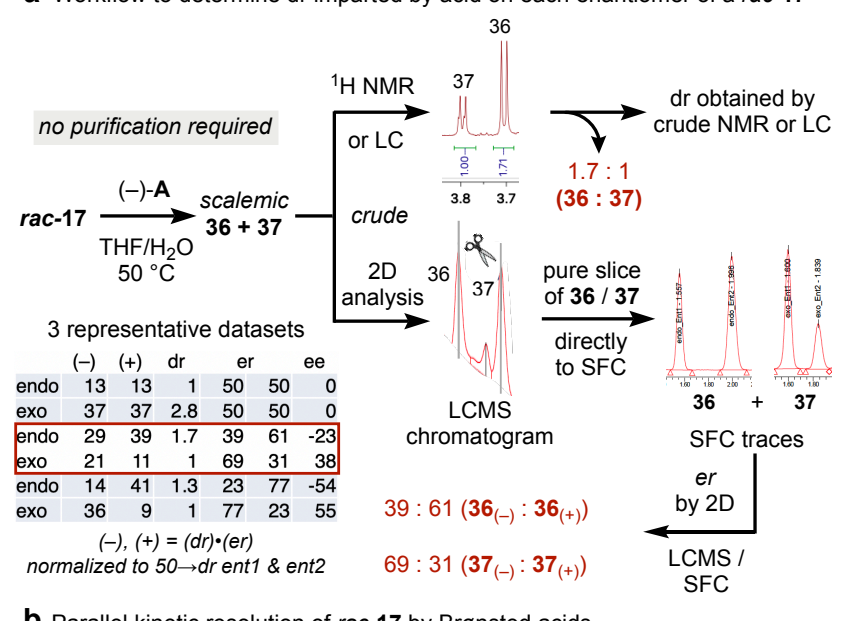

b Parallel kinetic resolution of rac-17 by Brønsted acids

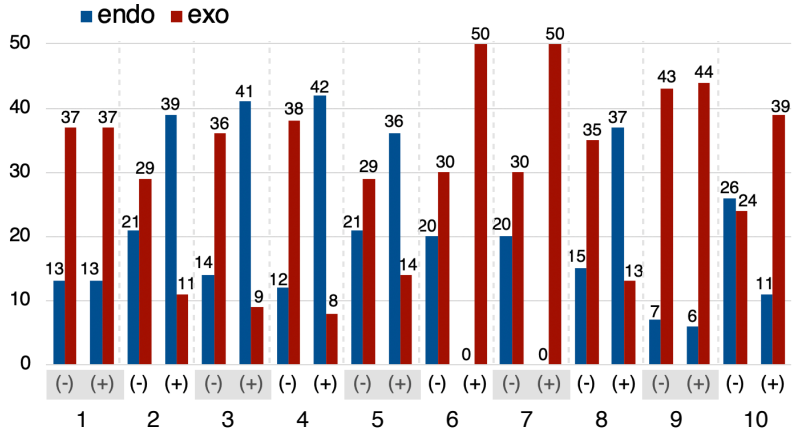

C Diastereoselectivity imparted by Brønsted acids using pure (+)-17

\begin{tabular}{clc} 
Entry & \multicolumn{1}{c}{ Conditions } & $\begin{array}{c}\mathbf{3 6}: \mathbf{3 7} \\
\text { from }(+)-\mathbf{1 7}\end{array}$ \\
\hline 1 & PTSA, THF/ $/ \mathrm{H}_{2} \mathrm{O}$, rt & $1.0: 2.8$ \\
2 & $(\mathrm{R})-\mathbf{A}, \mathrm{THF} / \mathrm{H}_{2} \mathrm{O}, 50{ }^{\circ} \mathrm{C}$ & $2.8: 1.0$ \\
3 & $(\mathrm{R})-\mathrm{A}, \mathrm{PhMe}, 23^{\circ} \mathrm{C}$ & $4.6: 1.0$ \\
\hline 4 & $(\mathrm{R})-\mathrm{A}, \mathrm{C}_{6} \mathrm{H}_{12}, 23^{\circ} \mathrm{C}$ & $5.3: 1.0$ \\
\hline 5 & $(\mathrm{R})-\mathrm{A}, \mathrm{DCM}, 23^{\circ} \mathrm{C}$ & $2.6: 1.0$ \\
6 & $(\mathrm{~S})-\mathrm{B}, \mathrm{C}_{6} \mathrm{H}_{12}, 23^{\circ} \mathrm{C}$ & $0.0: 50.0$ \\
7 & $(\mathrm{~S})-\mathrm{C}, \mathrm{C}_{6} \mathrm{H}_{12}, 23^{\circ} \mathrm{C}$ & $0.0: 50.0$ \\
8 & $(\mathrm{R})-\mathrm{C}, \mathrm{THF}^{\circ} / \mathrm{H}_{2} \mathrm{O}, 50^{\circ} \mathrm{C}$ & $2.8: 1.0$ \\
9 & $(\mathrm{~S})-\mathrm{D}, \mathrm{C}_{6} \mathrm{H}_{12}, 23^{\circ} \mathrm{C}$ & $1.0: 7.3$ \\
\hline 10 & $(\mathrm{~S})-\mathrm{E}, \mathrm{C}_{6} \mathrm{H}_{12}, 23^{\circ} \mathrm{C}$ & $1.0: 3.5$
\end{tabular}

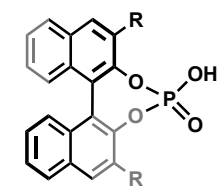

A: $R=9$-phenanthryl

B: $\mathrm{R}=2,4,6-\left(\mathrm{C}_{6} \mathrm{H}_{12}\right)_{3} \mathrm{Ph}$

C: $\mathrm{R}=2,4,6-(i-\mathrm{Pr})_{3} \mathrm{Ph}$

D: $\mathrm{R}=\mathrm{H}$

E: $\mathrm{R}=3,5-\left(\mathrm{CF}_{3}\right)_{2} \mathrm{Ph}$

Figure 17. Optimization of acetalization dr via parallel kinetic resolution (PKR): workflow and data analysis (SI22).

The data in Figure 17 indicated that lower temperature, nonaqueous solvent (cyclohexane) and phenanthryl substituents on the chiral acid provided the highest selectivity $(5.3: 1.0)$ for endo-isomer $(+)-36$ from $(+)-\mathbf{1 7}$, whereas the exo-isomer $(-$ $-)-37$ was produced from (-)-17 with $1.0: 3.2$ selectivity. Diastereoselectivity combined with product enantioenrichment allowed prediction of $\mathrm{dr}$ using a one enantiomer of $\mathbf{1 7}$. This singular antipode was available from the catalytic enantioselective Reformatsky discovered in step two of the synthesis, combined with purification by crystallization, which enriched enantiomeric excess to $>99 \%$ ee. In the event, $(+)-17$ was converted with $5.3: 1.0$ selectivity to $(+)-36$ under optimal conditions.

The alkyne subunit had been installed as a surrogate for an acetate appendage, however its hydration and oxidation proved non-trivial. We planned to oxidize the alkyne using standard conditions (Figure 18) ${ }^{78}$ Unfortunately, only hydroboration $\left(\mathrm{BH}_{3} \bullet \mathrm{THF}\right)$ and iterative oxidation $\left(1 . \mathrm{Na}_{2} \mathrm{~B}_{2} \mathrm{O}_{3} ; 2\right.$. TEMPO, PIDA) successfully generated the target lactone $\mathbf{4 8}$, and this only in $23 \%$ yield. The yields severely diminished when the reaction was run on scales larger than $0.1 \mathrm{mmol}$. An alternative solution was inspired by a report by Julia that alkyne oxidation could be carried out directly using LiOO $t$ $\mathrm{Bu} / \mathrm{LiHMDS}$ by way of an intermediate lithium ynolate. ${ }^{79} \mathrm{Ap}-$ plication of these conditions led to recovered starting material and a trace amount of the TMS-protected alkyne, which implicated at least partial deprotonation of $\mathbf{5}$. We hypothesized that the lithium acetylide could not efficiently coordinate LiOOt$\mathrm{Bu}$, whereas a more Lewis acidic substrate might. Ultimately, we found that the lithium acetylide intermediate reacted efficiently with trimethylborate, $\mathrm{B}(\mathrm{OMe})_{3}$ to generate a transient alkynylborate anion. Only $m$-CPBA effected oxidation to the lactone, presumably by protonation of one methoxide ligand and capture of the trivalent boron, ${ }^{80}$ as suggested by early work on alkoxyboronates by Brown. ${ }^{81}$ Migration of the alkyne into the adjacent $\mathrm{O}-\mathrm{O} \sigma^{*}$ orbital would generate an intermediate boron ynolate, which could cyclize to lactone 48 via several pathways. Protonation with benzoic acid or perbenzoic acid could generate a ketene or, if captured, a mixed anhydride. Alternatively, the boron ynolate might be stable until addition of aqueous $\mathrm{NH}_{4} \mathrm{Cl}$. No byproducts were isolated that provided insight into mechanistic details, but an upcoming report from the Ohtawa lab seeks to shed light on this question. ${ }^{82}$
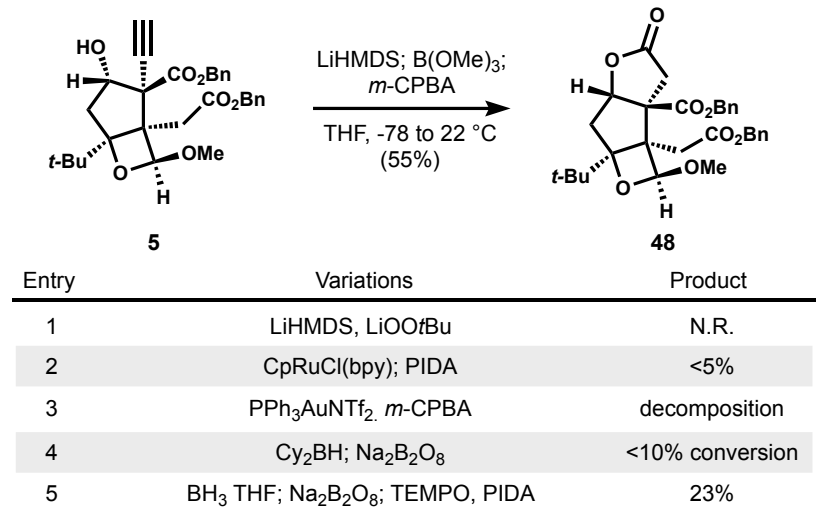

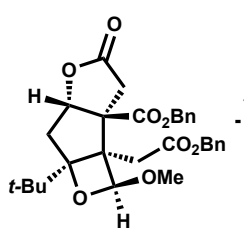

48: synthetic equivalent

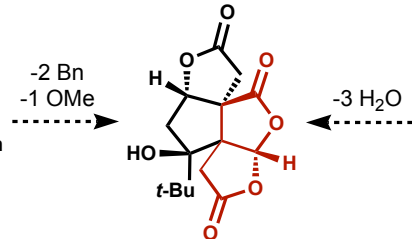

2: thermodynamic sink?

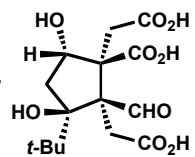

3: retrosythetic
Figure 18. Alkyne to lactone conversion via spborylation/oxidation versus other tactics. The resulting synthetic equivalent to $\mathbf{2}$ did not obviously resemble the retrosynthetic target.

The question of whether the bilobalide ring system represented a thermodynamic sink and whether the unusually stable oxetane would deprotect under suitable conditions now lay before us. Impatience to answer this question and attempt the 
final, deep oxidation ('inner' versus 'outer,' lactone D versus A) led to a mechanistic morass that would consume over ninemonths of research.

Because oxetane 48 proved stable to Brønsted acid, a dealkylating Lewis acid, $\mathrm{BBr}_{3}(>10$ equivalents), was added to 48 to decompose both the methoxy acetal and the benzyl esters. To our surprise, des-hydroxybilobalide was produced on the first attempt, albeit in apparent low (ca. 30\%) yield. This result dispelled the uncertainty of whether the bilobalide dilactone acetal would serve as a thermodynamic sink to which the acyclic carboxylates might funnel. Flush with success, the single milligram of crude $\mathbf{2}$ was subjected to conditions that might hydroxylate either lactone, with the hope that luck was now on our side. Treatment of crude $\mathbf{2}$ with NaHMDS, $\mathrm{P}(\mathrm{OEt})_{3}$, and $\mathrm{O}_{2}$ delivered a new product by TLC (with prolonged heating for visualization, see below) and ${ }^{1} \mathrm{H}$ NMR of material recovered after workup showed unambiguous peaks of bilobalide as the major product (see Figure 19a). ${ }^{83}$

a. enolate hydroxylation using crude 2 yielded bilobalide (1), but only once
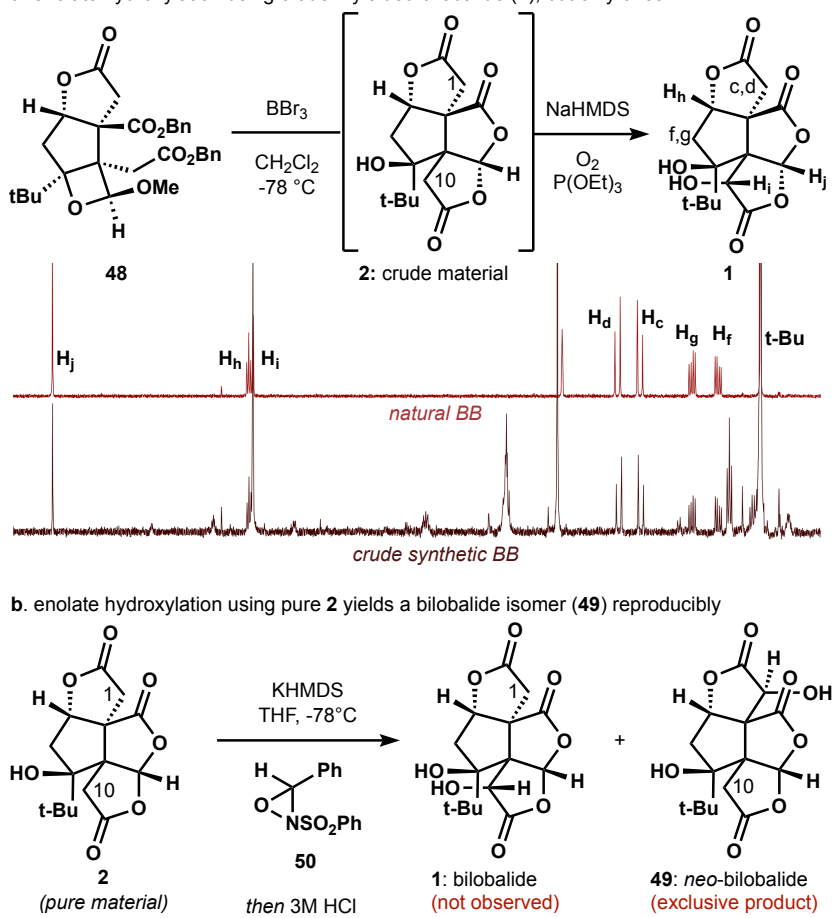

Figure 19a. $\mathrm{BBr}_{3}$ deprotection/ $\mathrm{O}_{2}$ oxidation of crude material delivered bilobalide (1) on the first attempt and $\mathbf{b}$. never again.

This result was extraordinary given the apparent inaccessibility of the $\mathrm{C} 10$ protons. Our delight was fleeting: the reaction has never been reproduced even to this day.

Repeated attempts to effect the oxidation of $\mathbf{2}$ to $\mathbf{1}$ under the original conditions by the same or different personnel either returned starting material or produced the undesired (but expected) isomer, designated neo-bilobalide (49, Figure 19b), a result of oxidizing the more accessible 'outer' lactone. Although the adage 'garbage-in, garbage-out' proved partly true, a reproducible procedure for the final oxidation may never have been discovered without this crucial reconnaissance. The process of invention now became archaeology: what had happened the first time? a. isomerization at cryogenic temperatures

observed by ${ }^{1} \mathrm{H}$ NMR
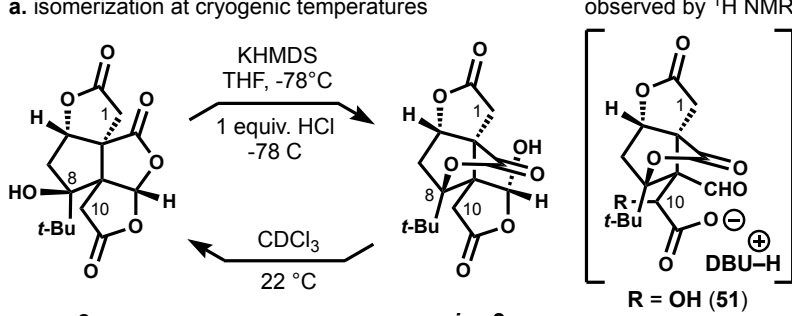

iso-2

b. isomerization appears to change inner lactone accessibility

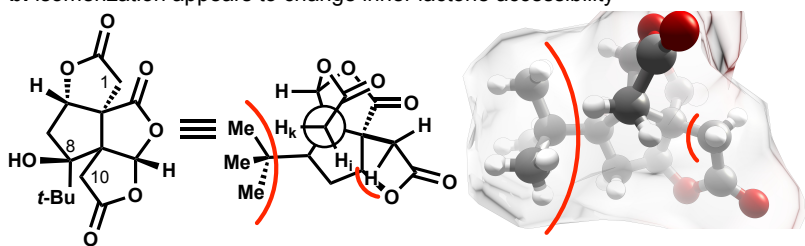

inner lactone protons $\left(H_{i}, H_{k}\right)$ inaccessible
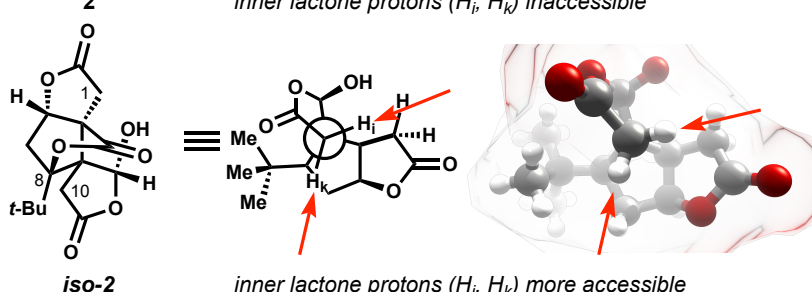

iso-

inner lactone protons $\left(H_{i}, H_{k}\right)$ more accessible

Figure 20. Does rearrangement to the iso-bilobalide skeleton allow access to the inner lactone protons $\mathrm{H}_{\mathrm{i}}$ and $\mathrm{H}_{\mathrm{k}}$ ? a. isomerization can be effected with strong base at low temperature or weak base at room temperature (quantitative in $10 \mathrm{~min}$.); b. isomerization changes proton accessibility on $180^{\circ}$ trajectories of approach.

The base lability of bilobalide provided the first evidence for a complex mechanism of oxidation. Its instability has led to many optimizations of extraction and purification from $G$. biloba leaves. ${ }^{84}$ At $\mathrm{pH} \mathrm{3}$, the trilactone fraction (ginkgolides and bilobalide) appears stable, but at $\mathrm{pH} 6.5$ mono-lactone saponification of ginkgolide B occurs. Strongly basic $(\mathrm{pH}$ 10.4) aqueous washes of crude $G$. biloba extract removes BB and ginkgolide $\mathrm{C}$ from the organic phase. However, acidification of the aqueous phase with $1 \mathrm{~N} \mathrm{HCl}$, followed by reextraction with EtOAc only leads to the recovery of ginkgolide $\mathrm{C}$ whereas $\mathrm{BB}$ decomposes and cannot be recovered. ${ }^{84 \mathrm{a}}$ $\mathrm{BB}$ has been found to irreversibly decompose at $\mathrm{pH}$ values above $7,{ }^{85}$ likely through irreversible lactone opening. ${ }^{86}$ To date, these decomposition byproducts have not been characterized and the pathway for irreversible $\mathrm{BB}$ decomposition remains unknown. This information is important for both isolation and pharmacokinetics: BB degrades in rat heparinized plasma $\left(k=0.5465 \mathrm{~h}^{-1}\right)$ and even in $\mathrm{pH} 7.4$ buffer $(k=0.1868$ $\left.\mathrm{h}^{-1}\right){ }^{87}$

The base instability of bilobalide might derive from its rapid rearrangement to iso-bilobalide. A similar rearrangement had been observed by Weinges and co-workers upon treatment of bilobalide with acetic anhydride and pyridine, which delivered diacetoxy-iso-bilobalide. ${ }^{88}$ However, we found rearrangement occurred simply upon treatment with base (Figure 20a). The C8 tertiary alcohol is positioned within the Burgi-Dunitz angle of lactone A, which is rendered more electrophilic by lone pair hyperconjugation, and its translactonization pulls the tert-butyl away from $\mathrm{C} 10$. For example, addition of 1 equivalent of the weak base $\mathrm{DBU}$ to a $\mathrm{CDCl}_{3}$ solution of 1 or 2 effects quantita- 
tive conversion to translactonized products $\mathbf{5 1}$ or $\mathbf{5 2}$. Similarly, we found that treatment of 2 at $-78^{\circ} \mathrm{C}$ with 1 equivalent of the strong base KHMDS - the same base used for attempted lactone hydroxylation-followed by 1 equivalent of $1 \mathrm{M}$ methanolic $\mathrm{HCl}$ quantitatively forms iso-2, which is unstable in acid, difficult to isolate and slowly reverts to $\mathbf{2}$. Since rearrangement to the iso-bilobalide skeleton (iso-2) occurred first, it clearly played a role in the irreproducible deep oxidation. a. Pd catalyzed hydrogenolysis is superior to $\mathrm{BBr}_{3}$ dealkylation
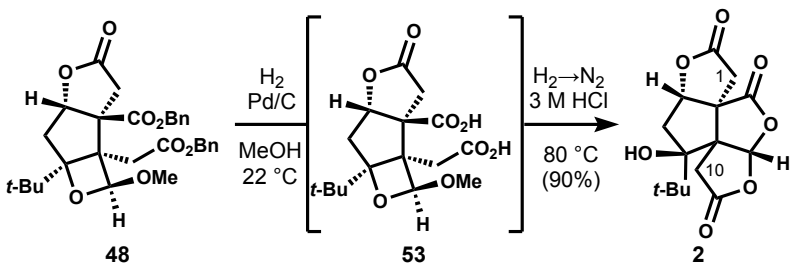

persistent in $3 \mathrm{M} \mathrm{HCl}$ reactive in $3 \mathrm{M} \mathrm{HCl}$

b. Boron Lewis acids do not affect oxidation regioselectivity, but a silicon group helps

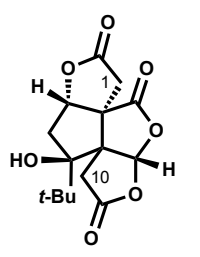

$\underset{22}{{ }^{\circ} \mathrm{C} \downarrow} \mathrm{CH}_{2} \mathrm{Cl}_{2} \downarrow \begin{aligned} & \text { TBSOTf, 2,6-lutidine } \\ & (100 \%)\end{aligned}$

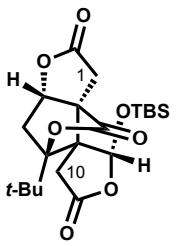

55

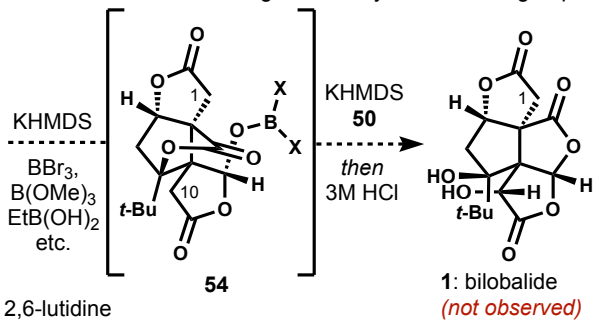

(not observed)

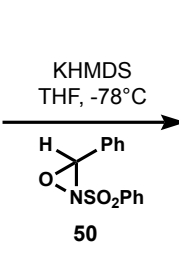

then $3 \mathrm{M} \mathrm{HCl}$

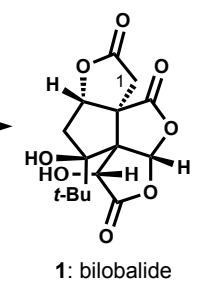

1

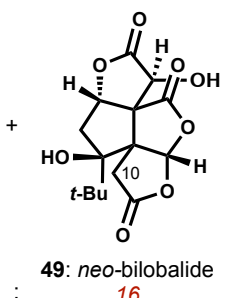

16
Figure 21a. Replacement of $\mathrm{BBr}_{3}$ in a global deprotection increases yield but removes the boron impurities that may have facilitated $\mathrm{C} 10$ hydroxylation; b. boron Lewis acids are ineffectual, but an isomerized silyl ether provides a trace of $\mathbf{1}$.

The original, successful oxidation ( 2 to 1) must have occurred via the base-induced iso-bilobalide rearrangement, given its facility in the presence of base. This hypothesis benefited from molecular models that indicated the iso-bilobalide skeleton increased access to $\mathrm{C} 10$ of the 'inner' lactone (see Figure 20b). However, if rearrangement alone were necessary, any successful oxidation should deliver 1 . In reality, any conditions for $\alpha$-hydroxylation including KHMDS/ Davis' oxaziridine (50) yielded exclusively the $\mathrm{C} 1$-oxidized isomer 49 via oxidation of the outer lactone.

Recall that global deprotection of $\mathbf{4 8}$ was carried out using $\mathrm{BBr}_{3}$. This procedure reliably provided low yields of $\mathbf{2}$, but when oxidation to $\mathbf{1}$ failed repeatedly (with crude or purified material), we took the opportunity to improve the deprotection. To our surprise, $\mathbf{4 8}$ was completely stable to $3 \mathrm{M} \mathrm{HCl}$ at $80{ }^{\circ} \mathrm{C}$ for over $12 \mathrm{~h}$, reflecting the remarkable 'corset' fortification of the oxetane acetal (see Figure 15). However, hydrogenolysis of the benzyl esters (Figure 21) significantly enhanced the reactivity of the oxetane: now $3 \mathrm{M} \mathrm{HCl}$ over $12 \mathrm{~h}$ produced 2 cleanly, which supports the equilibrium 'corset' effect proposed above.
Perhaps a boron impurity led to formation of 1 by capturing the opened isomer as 54, the borate of iso-2. Unfortunately, addition of boron Lewis acids- $\mathrm{BBr}_{3}, \mathrm{~B}(\mathrm{OMe})_{2}, \mathrm{EtB}(\mathrm{OH})_{2}$, etc.- - either prevented reaction or had no effect. However, attempts to mimic the borylated iso-bilobalide intermediate using a tert-butyl-dimethylsilyl (TBS) group (55) met with modest success: for the first time in nine months, bilobalide was observed in the crude ${ }^{1} \mathrm{H} \mathrm{NMR}$, albeit as the minor isomer (1:16) versus neo-bilobalide.
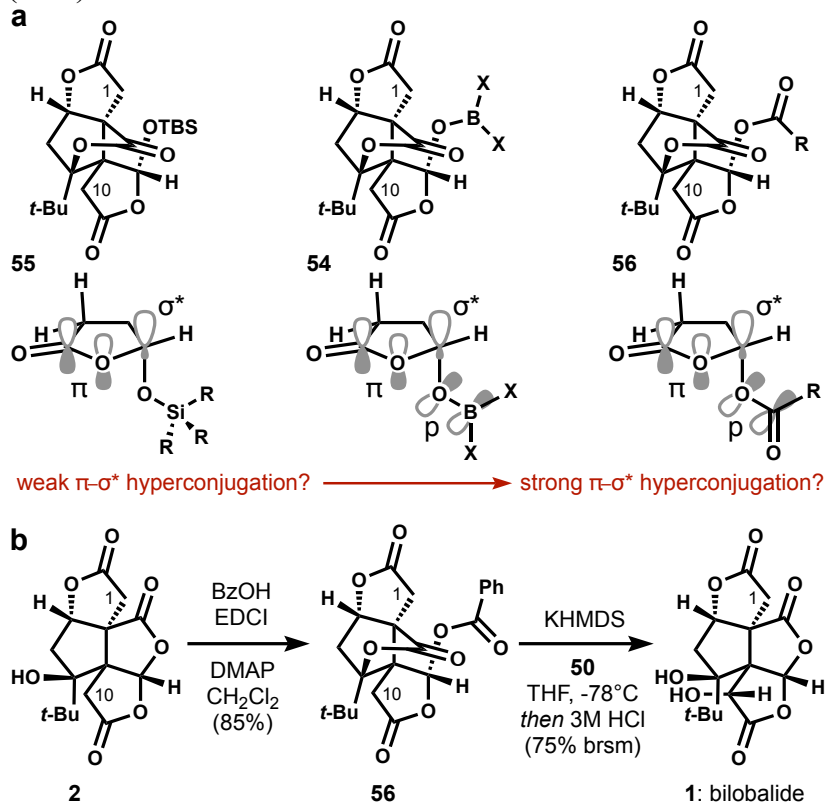

c

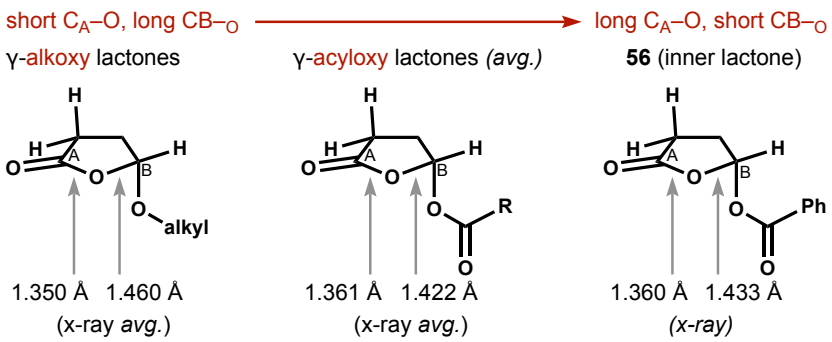

Figure 22a-c. Did hyperconjugation from an intermediate borate ester acidify the inner lactone? A stable benzoate ester causes selective oxidation of $\mathrm{C} 10$ to generate bilobalide (1).

A major difference between silicon and boron is the effect of valence bonding on electron delocalization. In this case, a trivalent boron (e.g. 54) might acidify the $\alpha$-protons (i.e. stabilize the corresponding enolate) via $\mathrm{B}-\mathrm{O} \pi$-bonding ${ }^{89}$ and delocalization of the lactone $\pi$-system into an adjacent $\mathrm{C}-\mathrm{O} \sigma^{*}$ orbital, more so than the corresponding $\mathrm{Si}-\mathrm{O}$ substituent (e.g. 55). Analogous delocalization is common in glycosides and reflected by changes in bond lengths to the anomeric carbon. ${ }^{90}$ In the case of chloro-glycosides, the axial conformer has a $0.62 \AA$ shorter $\mathrm{C}-\mathrm{O}$ bond and a $0.105 \AA$ longer $\mathrm{C}-\mathrm{Cl}$ bond than its equatorial isomer (see Supporting Information), as a result of oxygen lone-pair delocalization into the antiperiplanar antibonding orbital of the adjacent carbon halogen bond.. ${ }^{91}$ In the case of an $\alpha$-hydroxy lactone, this lone pair delocalization into the anomeric antibonding orbital could decrease the lone pair donation into the adjacent carbonyl, making it more electron deficient. ${ }^{92}$ In turn, this deficiency should make the 
carbonyl more ketone-like and result in acidification of the $\alpha$ protons.

We were unable to isolate a boric acid or boric ester derivative of des-hydroxy-isobilobalide iso-2, consistent with the absence of borylated $\gamma$-hydroxy lactones in the literature, so we turned to an electronically equivalent group. Whereas silylated $\gamma$-hydroxy lactone $\mathbf{5 5}$ would not benefit from the same acidification of a borylated $\gamma$-hydroxy lactone (cf. TBSOH $\mathrm{pKa}=15.36$ versus $\left.\mathrm{B}(\mathrm{OH})_{3} \mathrm{pKa}=9.24\right),{ }^{93}$ a benzoyl group $(\mathrm{BzOH} \mathrm{pKa}=4.20)$ might prove suitable. This derivative $(\mathbf{5 6})$ was synthesized from $\mathbf{2}$ with DMAP, EDCI and benzoic acid (see Figure 22a/b). Drawing analogy to haloglycosides (see above), we compared the bond lengths at the anomeric carbon of 56 to other substituted $\gamma$-hydroxy lactones (see Supporting Information S38) and observed a similar trend. Electron withdrawing groups adjacent to the ethereal oxygen of $\gamma$-hydroxy lactones cause a shortening of the anomeric $\mathrm{C}-\mathrm{O}$ bond and a lengthening of the lactone $\mathrm{C}-\mathrm{O}$ bond (see Figure 22c). These data derived from crystal structures, and DFT calculations found similar trends (Figure 22d). Compared to 4-hydroxy- $\gamma$ lactone $(\mathrm{pKa}=22)$ and its tert-butyldimethylsilyl ether (21.8), the borate (18.6), acetate (17.3) and benzoate (17.8) were significantly more acidic and exhibited bond length changes consistent with $\mathrm{O}$ lone pair delocalization into the $\sigma^{*}$ orbital of the substituent; overall this decrease in the $\mathrm{C}-\mathrm{O} \pi$ character might acidify the $\mathrm{C}$-10 $\alpha$-protons.

\begin{tabular}{lllllll}
$\mathbf{R}$ & $a$ & $b$ & $\Delta \mathrm{G}_{\text {depr }}$ & $\mathrm{pKa}$ & $\Delta \mathrm{pKa}$ \\
\cline { 2 - 7 } & $\mathrm{H}$ & 1.35 & 1.44 & 27.5 & 22 & 0 \\
$\mathrm{OTBS}$ & 1.36 & 1.44 & 27.2 & 21.8 & 0.2 \\
& $\mathrm{OT}(\mathrm{OH})_{2}$ & 1.36 & 1.43 & 23.2 & 18.6 & 3.4 \\
& $\mathrm{OCOMe}$ & 1.36 & 1.41 & 21.6 & 17.3 & 4.7 \\
& $\mathrm{OCOPh}$ & 1.37 & 1.41 & 22.2 & 17.8 & 4.2
\end{tabular}

Figure 22d. Calculated bond lengths (ångströms) and free energies of deprotonation $(\mathrm{kcal} / \mathrm{mol})$ by acetate anion at the $\omega B 97 X-D / 6-311++G(d, p), S M D(T H F) / / \omega B 97 X-D / 6-$ $31+\mathrm{G}(\mathrm{d}, \mathrm{p})$ level of theory.

In the event, subjection of benzoylated intermediate $\mathbf{5 6}$ to KHMDS and Davis oxaziridine yielded, to our immense relief, bilobalide (1) in 91:9 preference to its isomer, neo-bilobalide (49). This acidification of $\alpha$-protons through the anomeric effect appeared to be unique and warranted further investigation. Acidification of the $\alpha$-protons might open two pathways to $\mathrm{C} 10$ oxidation: 1) direct deprotonation of the $\mathrm{C} 10$ position $\left(\mathbf{5 6} \cdot K_{\mathbf{D}}\right.$, Figure 23) or 2) indirect deprotonation via proton exchange with the enolate of the more accessible outer lactone A $\left(\mathbf{5 6} \cdot K_{\mathbf{A}}\right)$.

Experiment effectively differentiated these pathways. First, we found that deprotonation occurred very quickly: incubation of 56 with base for 60,30 or 10 minutes did not change conversion to 1. Second, based on recent reports that Davis' oxaziridine (50) does not react with KHMDS at cryogenic temperatures, ${ }^{94}$ we probed the possibility of enolate-lactone proton exchange by addition of KHMDS to a mixture of $\mathbf{5 0}$ and $\mathbf{5 6}$. The result was a 60:40 mixture of 1:49 (bilobalide : neobilobalide), revealing that deprotonation occurs at $\mathrm{C} 1$ (lactone A, $\mathbf{5 6} \cdot \mathbf{K}_{\mathrm{A}}$ ) followed by an enolate equilibration to C10 in the anomerically-stabilized lactone D $\left(\mathbf{5 6} \cdot \mathbf{K}_{\mathbf{D}}\right)$. Whether this equilibration occurred by intermolecular or intramolecular proton transfer was determined by varying the concentration of $\mathbf{5 6}$ at a constant concentration of 50. A positive correlation between 1:49 and [50] revealed an intermolecular exchange: as the rate of productive collisions between substrates increased, the concentration of inner enolate increased.
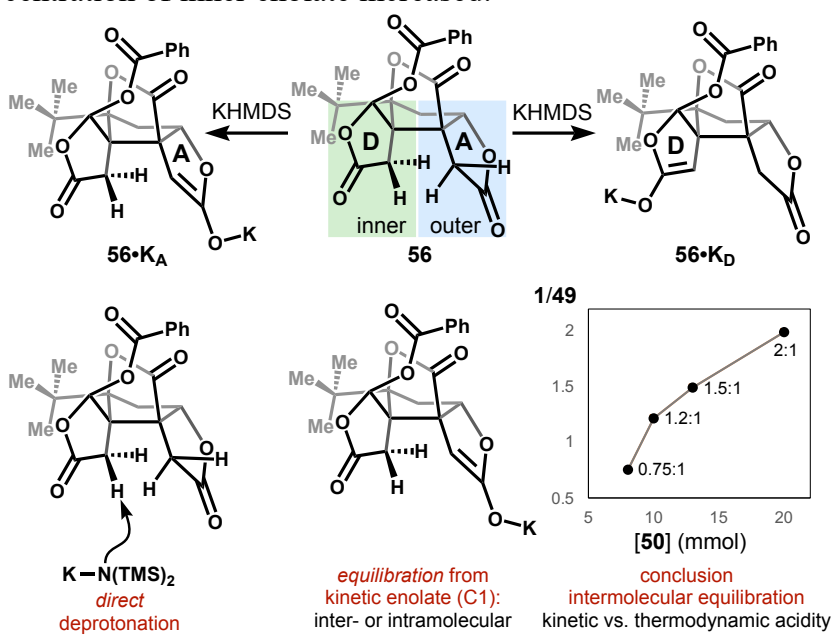

Figure 23. Addition of 56 (varying concentrations) to a solution of KHMDS and $\mathbf{5 0}$ (constant concentration) indicates intermolecular proton transfer and equilibration to a thermodynamic, inner enolate from the kinetic, outer enolate.

Consistent with this data, deprotonation at C10 (inner lactone) was calculated to be $4.3 \mathrm{kcal} / \mathrm{mol}$ more favorable than deprotonation at $\mathrm{C} 1$ (outer lactone), meaning that the inner lactone is about $3 \mathrm{pKa}$ units more acidic. This acidification is primarily due to the anomeric and inductive effects of the pendant ester moiety, which is absent in the outer lactone ring. ${ }^{95}$ We suspect that the original, irreproducible oxidation occurred from an iso-bilobalide borate (54, Figure 21a, 22a) similar to 56.

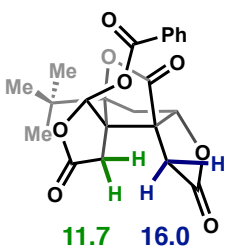

Figure 24. Calculated free energies $(\mathrm{kcal} / \mathrm{mol})$ of deprotonation by acetate anion at the $\omega \mathrm{B} 97 \mathrm{X}-\mathrm{D} / 6-311++\mathrm{G}(\mathrm{d}, \mathrm{p})$, $\mathrm{SMD}(\mathrm{THF}) / / \omega \mathrm{B} 97 \mathrm{X}-\mathrm{D} / 6-31+\mathrm{G}(\mathrm{d}, \mathrm{p})$ level of theory.

All the data taken together indicates that the inner lactone is inaccessible to base for direct deprotonation. Even in the unfolded iso-bilobalide analogs (55) and (56), deprotonation occurs initially from the outer lactone. Fortunately, acidification by the benzoate allows the outer lactone enolate-the kinetic acid - to undergo intermolecular proton transfer to the inner lactone - the thermodynamic acid. This funneling of reactivity from an outer to an inner site allows deep oxidation and completion of the target.

Conclusion. Chemical synthesis can be viewed as the encoding of information content in a molecular structure. Secondary metabolites tend to encode information differently than other types of complex structures (dendrimers, dyes, drugs) and include a greater fraction of $\mathrm{sp}^{3}$ hybridized atoms $\left(\mathrm{Fsp}^{3}\right)$, high chiral atom content, high heteroatom content and low aroma- 
ticity. Total synthesis encodes this complexity by joining together low information content reactants, but aims for greaterthan-additive products. Our group (R.M.D, M.A.B, M.O., R.A.S.) is interested in objectively measuring this process and has turned to Böttcher's complexity index $\left(\mathrm{C}_{\mathrm{m}}\right)^{25}$ to formalize intrinsic information content. ${ }^{96}$ Böttcher scores assign information content (mcbits) to each non-hydrogen atom, which are added to measure molecular complexity, a comparative index given as $C_{m}$. Representative Böttcher indices correspond to the results of Bertz's graph theory-based $C(\eta, \varepsilon)$ complexity index, yet can be done with pencil and paper, albeit slowly. The Supporting Information for this paper includes a Python script (written by S. F.) that annotates molecules with Böttcher scores using a SMILES string input at a rate of $c a$. 132,000 compounds per minute, simplifying use of this metric. $C_{m}$ does not include any aspect of synthetic difficulty, a changing and subjective measure. ${ }^{97}$ Its ease and objectivity make $C_{m}$ an appealing measurement of complexity.

In Figure 25, we view the three syntheses of bilobalide through the lens of $\mathrm{C}_{\mathrm{m}}$ changes. Each intermediate was assigned a $C_{m}$ value and scores were averaged per atom to mitigate the effect of protecting groups, which can carry high information content without contributing to core complexity. After normalizing the $\mathrm{C}_{\mathrm{m}}$ scores, all three syntheses started from similar complexity scores, but advanced to the target at different rates and different sacrifices in complexity, reflecting different step counts.

We had qualitatively rationalized our choices in synthesis design as efficient through minimization of redox reactions and sought a Böttcher analysis to quantitatively interrogate this idea. Summation of the raw scores of carbons that have an oxygen atom in bilobalide $(\mathrm{C} 2,4,6,8,10,11,12)$ revealed three trends: 1) our work began from a higher total score, largely due to initial incorporation of bislactone oxygens in their final oxidation state, and therefore minimized the total oxidative distance to product, significantly lowering necessary operations; 2) the percentage of steps in a synthesis that result in an overall decrease in complexity at the carbons of interest was generally consistent across all three syntheses regardless of step count: this work: $25 \%$, Crimmins: $35 \%$, Corey: $27 \%$, therefore future strategies might try to minimize these sacrificial maneauvers; 3) a direct correlation existed between step count and complexity score changes at carbons bearing a heteroatom in a particular synthesis: this work $=10(83 \%$ of 12 total steps), Crimmins $=16$ (94\% of 17 total steps, racemic), and Corey $=20$ ( $83 \%$ of 24 total steps, asymmetric). The most telling metric of synthetic efficiency here is the total number of times these oxidized carbon atom change complexity scores throughout the synthesis. Ideally, a carbon atom is introduced in the synthesis at its final value (oxidation state, hybridization, stereochemistry) or undergoes minimal transformations to reach the target score.

Information content is easy to buy; information density is not. The dense packaging of information in natural product space is not unique to bilobalide; in fact, high information density may describe much of natural product space. Bilobalide uniquely fills a biologically-privileged and poorlyexplored region of chemical space characterized by unusually information-dense structures. Our synthesis addresses the challenge of encoding dense content through several features:
1) embedding oxygenation, largely of the correct oxidation state, in starting materials; 2) development of a catalytic, asymmetric Reformatsky reaction to merge these starting materials with high enantioselectivity; 3 ) use of radical reactions that benefit from early transition states to establish sterically congested centers, including 4) a solvent-controlled stereoselective Mukaiyama hydration that tolerates steric bulk via an outer-sphere MHAT elementary step; 5) use of extreme steric repulsion to leverage an unusually stable oxetane acetal in stereoselective $\mathrm{C}-\mathrm{C}$ bond formation via 6 ) the sterically small, but highly electrophilic Waser's reagent (TMS-EBX). Finally, the hypothesis that the bilobalide scaffold represented a thermodynamic sink allowed a late-stage, global deprotection to reach penultimate intermediate des-hydroxy-bilobalide (2). Crucial reconnaissance revealed the potential to effect selective oxidation of the hindered, inner lactone, which was reproducibly executed after significant interrogation and mechanism-based experimentation.
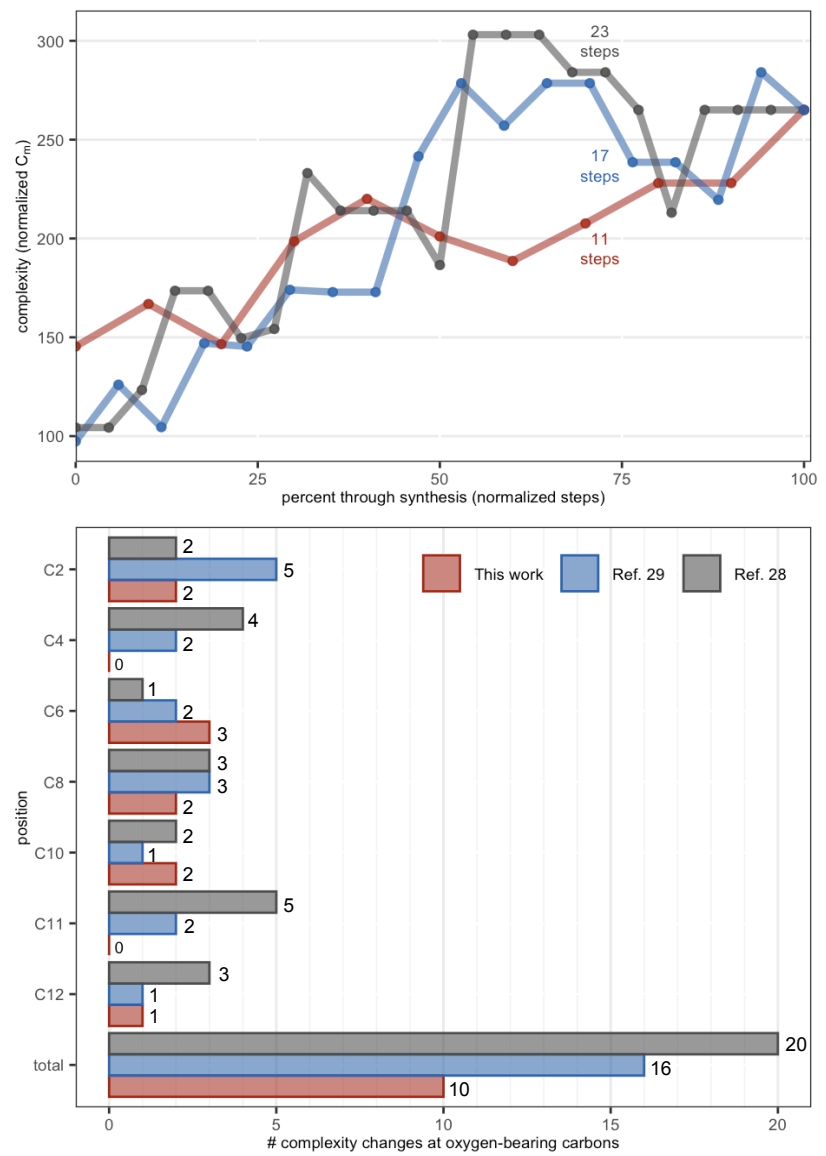

Figure 25. Different uses of Böttcher scores to annotate changes in complexity over the course of synthesis. a. Overshooting complexity is correlated to longer syntheses; b. number of complexity changes at each oxidized carbon is directly related to number of total steps.

Information dense (high mcbits/ $\AA^{3}$ ) natural products can be efficiently 'encoded' by incorporation of heteroatoms and oxidation states into starting materials. In principle and in practice, ${ }^{24}$ this approach allows a simple, combinatorial route to dense, polyoxygenated libraries. When paired with a macromolecular target, we imagine that oxygenation patterns of 
bioactive natural products can be altered to uncover simpler paths to oxygenated starting materials yet maintain target affinity. ${ }^{98,99}$ Both strategies require close collaboration between chemistry, computation and biology, and for the fields to understand one another with clarity. The push to develop retrosynthetic software superior to original iterations ${ }^{100}$ requires a clear-eyed understanding of how synthesis is actually conceived and executed. Here we have delineated in granular detail the recursive feedback of experimental results into synthetic design. In addition to the provision of tools for $\mathrm{GABA}_{\mathrm{A}} \mathrm{R}$ biology, we hope this work provides a clear illustration of the interplay between synthesis, mechanism and computation in the current era.

\section{ACKNOWLEDGMENT}

Support was provided by the National Institutes of Health (R35 GM122606; R.A.S.), the Uehara Memorial Foundation (M. O.) and the National Science Foundation (CHE-1764328; K.N.H). Calculations were performed on the Hoffman2 cluster at the University of California, Los Angeles, and the Extreme Science and Engineering Discovery Environment (XSEDE), supported by the National Science Foundation (grant OCI-1053575). C.C.L. is grateful to the Imperial College Bursary Scheme for funding. We thank Dr. Jason S. Chen and Brittany Sanchez of the Scripps Automated Synthesis Facility for help with separations and analysis.

\section{ASSOCIATED CONTENT}

\section{Supporting Information}

The Supporting Information is available free of charge on the ACS Publications website.

Experimental materials and methods; computational methods; Xray crystallography data; python script for Böttcher analysis

\section{AUTHOR INFORMATION}

\section{Corresponding Author}

*rshenvi@scripps.edu

\section{Present Addresses}

$†$ Graduate School of Pharmaceutical Sciences, Kitasato University, Tokyo, Japan

\section{Author Contributions}

$\$$ These authors contributed equally.

\section{REFERENCES}

(1) Seeff, L.; Bonkovsky, H. L.; Navarro, V. J.; Wang, G. Herbal products and the liver: A review of adverse effects and mechanisms. Gastroenterology 2015, 148, 517.

(2) https://www.fda.gov/food/dietary-supplements

(3) For modest, positive effects, see: (a) Le Bars, P. L.; Katz M. M.; Bermann N.; Itil, T. M.; Freedman, A. M; Schatzberg, A. F. A placebo-controlled, double-blind, randomized trial of an extract of Ginkgo biloba for dementia, North American EGb Study Group. JAMA 1997, 278, 1327; (b) Herrschaft, H.; Nacu, A.; Likhachev, S.; Sholomov, I.; Hoerr, R.; Schlaefke, S. Ginkgo biloba extract EGb 761® in dementia with neuropsychiatric features: a randomised, placebo-controlled trial to confirm the efficacy and safety of a daily dose of $240 \mathrm{mg}$. J. Psychiatr. Res. 2012, 46, 716; (c) Tan, M. S.; Yu, J. T.; Tan, C. C.; Wang, H. F.; Meng, X. F.; Wang, C.; Jiang, T.; Zhu, X.C.; Tan, L. Efficacy and adverse effects of ginkgo biloba for cognitive impairment and dementia: a systematic review and meta-analysis. J. Alzheimers Dis. 2015, 43, 589; (d) Yuan, Q.; Wang, C.W.; Shi, J.; Lin, Z. X. Effects of Ginkgo biloba on dementia: An overview of systematic reviews. J. Ethnopharmacol. 2017, 195, 1. For no effect, see: (e) Schneider, L.S.; DeKosky, S.T.; Farlow, M.R.; Tariot. P.N.; Hoerr, R.; Kieser, M. A randomized, double-blind, placebocontrolled trial of two doses of Ginkgo biloba extract in dementia of the Alzheimer's type. Curr Alzheimer Res. 2005, 5, 541; (f) Butler, M.; Nelson, V. A.; Davila, H.; Ratner, E.; Fink, H. A.; Hemmy, L. S.; McCarten, J. R.; Barclay, T. R.; Brasure, M.; Kane, R. L. Over-the-Counter Supplement Interventions to Prevent Cognitive Decline, Mild Cognitive Impairment, and Clinical Alzheimer-Type Dementia: A Systematic Review. Ann. Intern. Med. 2018, 168, 52; (g) DeKosky, S. T.; Williamson, J. D.; Fitzpatrick, A. L.; Kronmal, R. A.; Ives, D. G.; Saxton, J. A.; Lopez, O. L.; Burke, G.; Carlson, M. C.; Fried, L. P.; Kuller, L. H.; Robbins, J. A.; Tracy, R. P.; Woolard, N. F.; Dunn, L.; Snitz, B. E.; Nahin, R. L.; Furberg, C. D. Ginkgo Evaluation of Memory (GEM) Study Investigators, Ginkgo biloba for prevention of dementia: a randomized controlled trial. JAMA 2008, 300, 2253; (h) Vellas, B.; Coley, N.; Ousset, P. J.; Berrut, G.; Dartigues, J. F.; Dubois, B.; Grandjean, H.; Pasquier, F.; Piette, F.; Robert, P.; Touchon, J.; Garnier, Mathiex-Fortunet, H.; Andrieu, S.; GuidAge Study Group. Long-term use of standardised Ginkgo biloba extract for the prevention of Alzheimer's disease (GuidAge): a randomised placebo-controlled trial. Lancet Neurol. 2012, 10, 851; (i) Charemboon, T.; Jaisin, K. Ginkgo biloba for prevention of dementia: a systematic review and meta-analysis. J. Med. Assoc. Thai. 2015, 5, 508; (j) Laws, K. R.; Sweetnam, H.; Kondel, T. K. Is Ginkgo biloba a cognitive enhancer in healthy individuals? A meta-analysis. Hum Psychopharmacol. 2012, 6, 527; (k) For a summary, see: Nguyen, T.; Alzahrani, T. Ginkgo Biloba. [Updated 2019 Oct 3]. In: StatPearls [Internet]. Treasure Island (FL): StatPearls Publishing; 2019 Jan-. Available from: https://www.ncbi.nlm.nih.gov/books/NBK541024/

(4) Mei, N.; Guo, X.; Ren, Z.; Kobayashi, D.; Wada, K.; Guo, L. Review of Ginkgo biloba-induced toxicity, from experimental studies to human case reports. J. Environ. Sci. Health Part C 2017, 35, 1.

(5) Clarke, T. C.; Black, L. I.; Stussman, B. J.; Barnes, P. M.; Nahin, R. L. Trends in the use of complementary health approaches among adults: United States, 2002-2012. National health statistics reports 2015, no. 79. Hyattsville, MD: National Center for Health Statistics.

(6) Madgula V. L.; Avula, B.; Yu, Y. B.; Wang, Y. H.; Tchantchou, F.; Fisher, S.; Luo, Y.; Khan, I. A.; Khan, S. I. Intestinal and blood-brain barrier permeability of ginkgolides and bilobalide: in vitro and in vivo approaches. Planta Med. 2010, 76, 599.

(7) NTP Technical Report on the Toxicology and carcinogenesis studies of Ginkgo bilobaextract (CAS No. 90045-36-6) in F344/N rats and B6C3F1/N mice (Gavage studies). Natl. Toxicol. Program Tech. Rep.Ser. 2013, 578, 1.

(8) Fernandez, F.; Morishida, W.; Zuniga, E.; Nguyen, J.; Blank, M.; Malenka, R. C.; Garner, C. C. Pharmacotherapy for 
cognitive impairment in a mouse model of Down syndrome. $\mathrm{Na}$ ture Neurosci. 2007, 10, 411.

(9) Kiewert, C.; Kumar, V.; Hildmann, O.; Rueda, M.; Hartmann, J.; Naik, R. S.; Klein, J. Role of GABAergic antagonism in the neuroprotective effects of bilobalide. Brain Res. 2007, 1128, 70 .

(10) Masiulis, S. Desai, R.; Uchanski, T.; Martin, I. S.; Laverty, D.; Malinauskas, T.; Zivanov, J.; Pardon, E.; Kotecha, A.; Steyaert, J.; Miller, K. W.; Aricescu, A. R. GABAA receptor signaling mechanisms revealed by structural pharmacology. $\mathrm{Na}$ ture, 2019, 565, 454.

(11) (a) Atzori, C.; Bruno, A.; Chichino, G.; Bombardelli, E.; Scaglia, M.; Ghione, M. Activity of Bilobalide, a Sesquiterpene from Ginkgo biloba, on Pneumocystis carinii. Antimicrob. Agents Chemother. 1993, 37, 7, 1492; (b) Bombardelli, E.; Ghione, M. Pharmaceutical Uses of Bilobalide and Derivatives Thereof and Pharmaceutical Compositions Adapted for Such Use. U.S. Pat. Appl. US005264216A, 1993; (c) Bombardelli, E.; Ghione, M. Bilobalide Derivatives, Their Applications and Formulations Containing Them. US005202313A, 1993. (d) Bombardelli, E.; Ghione, M. Use of bilobalide and derivatives thereof for treating an infection in an individual and pharmaceutical compositions adapted for such use. EP0556051B1, 1997.

(12) (a) Pan, L.; Ren, L.; Chen, F.; Feng, Y.; Luo, Y. Antifeedant Activity of Ginkgo biloba Secondary Metabolites against Hyphantria cunea Larvae: Mechanisms and Applications. PLoS ONE 2016, 11, 1; (b) Pszczolkowski, M. A.; Durden, K.; Sellars, S.; Cowell, B.; Brown, J. J. Effects of Ginkgo biloba Constituents on Fruit-Infesting Behavior of Coding Moth (Cydia pomonella) in Apples. J. Agric. Food Chem. 2011, 59, 10879-10886. (c) Ahn, Y. J.; Kwon, M.; Park, H. M., Han, C. K. Potent Insecdticidal Activity of Ginkgo biloba Derived Trilactone Terpenes. ACS Symposium Series 1997, Ch. 7, 90; (d) Ozoe, Y. $\gamma$-aminobutyrateand glutamate-gated chloride channels as targets of insecticides. Advances in Insect Physiology, 2013, 44, 211.

(13) Major, R. T. The Ginkgo, the most ancient living tree, Science 1967, 157, 1270-1273.

(14) Koch, E. Inhibition of platelet activating factor (PAF)induced aggregation of human thrombocytes by ginkgolides: considerations on possible bleeding complications after oral intake of Ginkgo biloba extracts. Phytomedicine 2005, 12, 10 .

(15) Lee, D.; Su, H.; O’Dowed, D. K. GABA receptors Containing Rdl Subunits Mediate Fast Inhibitory Synaptic Transmission in Drosophola Neurons. J. Neuroscience, 2003, 23, 4625.

(16) (a) Constant, R. H. F.; Steichen, J. C.; Rocheleau, T. A.; Aronstein, K.; Roush, R. T. A single-amino acid substitution in a $\gamma$-aminobutyric acid subtype A receptor locus is associated with cyclodiene insecticide resistance in Drosophila populations. PNAS, 1993, 90, 1957; (b) Thompson, A. J.; McGonigle, I.; Duke, R.; Johnston, G. A. R.; Lummis, S. C. R. A single amino acid determines the toxicity of Ginkgo biloba extracts. FASEB J. 2012, $26,1884$.

(17) Ng, C. C.; Duke, R. K.; Hinton, T.; Johnston G. A. R. $\mathrm{GABA}_{\mathrm{A}}$ receptor cysteinyl mutants and the ginkgo terpenoid lactones bilobalide and ginkgolides. Eur. J. Pharmacol. 2016, 777, 136.

(18) Kalueff, A. V. Mapping convulsants' binding to the GABA-A receptor chloride ionophore: A proposed model for channel binding sites Neurochem. Int. 2007, 57, 61 .

(19) A note on nomenclature: residues in the transmembrane domain (TMD)-spanning $\alpha$-helices (see Figure 1) are numbered from 0', a conserved charged residue near the cytoplasm. Negative numbered residues (e.g. -2') are farther from the extracellular domain and positive numbered residues are closer. See Ref. 16b.

(20) Ng, C. C.; Duke, R. K.; Hinton, T.; Johnston, G. A. R. Effects of Bilobalide, Ginkgolide B and Picrotoxinin on GABA receptor Modulation by Structurally Diverse Positive Modulators. Eur. J. Pharmacol. 2017, 806, 83.

(21) (a) Jorgensen, J. L. Aldrin and Dieldrin: A Review of Research on Their Production, Environmental Deposition and Fate, Bioaccumulation, Toxicology, and Epidemiology in the United States. Environ. Health Perspect. 2001, 109, 113. (b) Agency for Toxic Substances and Disease Registry (ATSDR). 2002. Toxicological profile for Aldrin/Dieldrin. Atlanta, GA: U.S. Department of Health and Human Services, Public Health Service. (c) Soto, A. R.; Deichman W.B.; Major Metabolism and Acute Toxicity of Aldrin, Dieldrin, and Endrin. Environmental Research 1967, 1, 307.

(22) (a) Lu, H. H.; Martinez, M. D.; Shenvi, R. A. An eightstep- gram-scale synthesis of (-)-jiadifenolide. Nature Chem. 2015, 7, 604; (b) Ohtawa, M.; Krambis, M. J.; Cerne, R.; Schkeryantz, J. M.; Witkin, J. M., Shenvi, R. A. Synthesis of (-)11-O-Debenzoyltashironin: Neurotrophic Sesquiterpenes Cause Hyperexcitation. J. Am. Chem. Soc. 2017, 139, 9637.

(23) Witkin et. al. Pharmacological characterization of the neurotrophic sesquiterpenejiadifenolide reveals a non-convulsant signature and potential forprogression in neurodegenerative disease studies. Biochem. Pharmacol. 2018, 155, 61.

(24) Huffman, B. J.; Chen, S.; Schwarz, J. L; Plata, R. E.; Chin, E.; Houk, K. N.; Lairson, L. L.; Ryan A. Shenvi Electronic Complementarity Permits Hindered Butenolide Heterodimerization and Discovery of Novel cGAS/STING Pathway Antagonists, Nature Chem. 2020, 12, 310.

(25) Böttcher, T. An Additive Definition of Molecular Complexity. J. Chem. Inf. Model. 2016, 56, 462.

(26) Herzon, S. B. Emergent Properties of Natural Products. SynLett 2018, 29, 1823.

(27) Weir, C. J.; Ling, A. T. Y.; Belelli, D.; Wildsmith, J. A. W.; Peters, J. A.; Lambert, J. J. The interaction of anaesthetic steroids with recombinant glycine and $\mathrm{GABA}_{\mathrm{A}}$ receptors. $\mathrm{Br} . J$. Anaesth. 2004, 92, 704.

(28) (a) Corey, E. J.; Guo, S. Total synthesis of a C15 ginkgolide, ( \pm )-bilobalide. J. Am. Chem. Soc. 1987, 109, 7534-7536. (b) Corey, E. J.; Su, W. Enantioselective Total Synthesis of Bilobalide, A C15 Ginkgolide. Tetrahedron Lett. 1988, 29, 3423.

(29) Crimmins, M. T.; Jung, D. K.; Gray, J. L. Synthetic Studies on the Ginkgolides: Total Synthesis of $( \pm)$-Bilobalide. $J$. Am. Chem. Soc. 1993, 115, 3155.

(30) Normalization by dividing by atom count mitigates the influence of protecting groups which often encode large amounts of information that is irrelevant to building target complexity.

(31) Baker, M. A.; Demoret, R. M.; Ohtawa, M.; Shenvi, R. A. Concise asymmetric synthesis of (-)-bilobalide. Nature 2019, 575,643 .

(32) Schuster, D. I.; Lem, G.; Kaprinidis, N. A. New Insights into an old Mechanism: [2+2] Photocycloadditions of Enones to Alkenes. Chem. Rev. 1993, 93, 3 .

(33) (a) Iwasaki, K.; Wan, K. K.; Oppedisano, A.; Crossley, S. W. M.; Shenvi R. A. Simple, Chemoselective Hydrogenation with Thermodynamic Stereocontrol. J. Am. Chem. Soc. 2014, 136, 1303; b) Crossley, S. W. M.; Barabé, F.; Shenvi, R. A. Simple, 
Chemoselective, Catalytic Olefin Isomerization, J. Am. Chem. Soc. 2014, 136, 16788.

(34) Crossley, S. W. M.; Martinez, R. M.; Obradors, C.; Shenvi, R. A. Mn, Fe, and Co-Catalyzed Radical Hydrofunctionalizations of Olefins, Chem. Rev. 2016, 116, 8912.

(35) Evans, D. A.; Tedrow, J. S.; Shaw, J. T.; Downey, C. W. Diastereoselective Magnesium Halide-Catalyzed anti-Aldol Reactions of Chiral N-Acyloxazolidinones. J. Am. Chem. Soc. 2002, 124, 392.

(36) Nicolaou, K. C.; Ellery, S. P.; Chen, J. S. Samarium Diiodide-Mediated Reactions in Total Synthesis. Angew. Chem. Int. Ed. 2009, 48, 39, 7140 .

(37) Thankachan, A. P.; Asha, S.; Singhu, K. S.; Anilkumar, $\mathrm{G}$. An overview of $\mathrm{Zn}$-catalyzed enantioselective aldol type C-C bond formation. RSC Adv. 2015, 5, 62179.

(38) Kern, N.; Plesniak, M. P.; McDouall, J. J. W.; Procter, D. J. Enantioselective cyclizations and cyclization cascades of samarium ketyl radicals. Nature Chem. 2017, 9, 1198.

(39) Ribeiro, C. M. R.; Cordeiro de Farias, F. M. Chiral Ligands in the Asymmetric Reformatsky Reaction. Mini-Reviews in Organic Chemistry. 2006, 3, 1.

(40) Ojida, A.; Yamano, T.; Taya, N.; Tasaka, A. Highly Enantioselective Reformatsky Reaction of Ketones: ChelationAssisted Enantioface Discrimination. Org. Lett. 2002, 4, 18, 3051.

(41) Kloetzing, R. J.; Thaler, T.; Knochel, P. An Improved Asymmetric Reformatsky Reaction Mediated by (-)- $N, N-$ Dimethylaminoisoborneol. Org. Lett. 2006, 8, 1125.

(42) Wolf, C.; Moskowitz, M. Bisoxazolidine-Catalyzed Enantioselective Reformatsky Reaction. J. Org. Chem. 2011, 76, 6372.

(43) Fernández-Ibáñez, M. A.; Maciá, B.; Minnaard, A. J.; Feringa, B. L. Catalytic Enantioselective Reformatsky Reaction with Aldehydes. Angew. Chem.Int. Ed. 2008, 47, 1317.

(44) Lin, N.; Chen, M-M.; Luo, R-S.; Deng, T-Q.; Lu, G. Indolinylmethanol catalyzed enantioselective Reformatsky reaction with ketones. Tetrahedron: Asymm. 2010, 21, 2816.

(45) Cozzi, P. G.; Mignogna, A.; Zoli, L. Catalytic enantioselective Reformatsky reactions. Pure Appl. Chem. 2008, 80, 891.

(46) Kanai, K.; Wakabayashi, H.; Honda, T. RhodiumCatalyzed Reformatsky-Type Reaction. Org. Lett. 2000, 2, 2549.

(47) Li, Y.; He, B. Asymmetric Reformatsky Reaction of Aldehydes Catalyzed by Novel $\beta$-amino Alcohols and Zinc Complexes. Synth. Commun. 2014, 44, 13, 1938.

(48) Fornalczyk, M.; Singh, K.; Stuart, A. M. Enantioselective Reformatsky reaction of ethyl iododifluoroacetate with ketones. Org. Biomol. Chem. 2012, 10, 3332.

(49) Fernández-Ibáñez, M. A.; Maciá, B.; Alonso, D. A.; Pastor, I. M. Recent Advances in the Catalytic Enantioselective Reformatsky Reaction. Eur. J. Chem. 2013, 7028.

(50) Choppin, S.; Ferreiro-Medeiros, L.; Barbarotto, M.; Colobert, F. Recent advances in the diastereoselective Reformatsky-type reaction. Chem. Soc. Rev. 2013, 42, 937.

(51) Li, F.; Castle, S. L. Synthesis of the Acutumine Spirocycle via a Radical-Polar Crossover Reaction. Org. Lett. 2007, 9, 4033.

(52) Va, P.; Campbell, E. L.; Robertson, W. M.; Boger, D. L. Total Synthesis and Evaluation of a Key Series of C5-Substituted Vinblastine Derivatives. J. Am. Chem. Soc. 2010, 132, 8489.
(53) Schröder, M. Osmium Tetraoxide Cis Hydroxylation of Unsaturated Substrates. Chem. Rev. 1980, 80, 187.

(54) (a) Abelman, M. M.; Oh, T.; Overman, L. E. Intramolecular Alkene Arylations for Rapid Assembly of Polycyclic Systems Containing Quaternary Centers. A New Synthesis of Spirooxindoles and Other Fused and Bridged Ring Systems. J. Org. Chem. 1987, 52, 4130; (b) Ludlow, B. S.; Danielson, J.; Somfai, P. Comino Carbopalladation-Carbonylation: Investigation Substrate Scope. Adv. Synth. Catal. 2012, 354, 206; (c) Liu, P.; Seo, J. H.; Weinreb, S. M. Total Synthesis of the Polycyclic Fungal Metabolite ( \pm -Communesn F. Angew. Chem. Int. Ed. 2010, 49, 2000.

(55) Green, S. A.; Crossley, S. W. M.; Matos, J. L. M.; Vásquez-Céspedes, S.; Shevick, S.; Shenvi, R. A. The High Chemofidelity of Metal-Catalyzed Hydrogen Atom Transfer. Acc. Chem. Res. 2018, 51, 2628.

(56) Yaremenko, I. A.; Vil', V. A.; Demchuk, D. V.; Terent'ev, A. O. Rearrangements of organic peroxides and related processes. Beilstein J. Org. Chem. 2016, 12, 1647.

(57) Nishinaga, A.; Yamada, T.; Fujisawa, H.; Ishizaki, K.; Ihara, H.; Matsuura, T. Catalysis of Cobalt-Schiff Base Complexes In Oxygenation of Alkenes: On the Mechanism of Ketonization J. Mol. Catal. 1988, 48, 249.

(58) Only recently has absolute stereocontrol emerged. See: (a) Discolo, C. A.; Touney, E. E.; Pronin, S. V. J. Am. Chem. Soc. 2019, 44, 17527; (b) Ebisawa, K.; Izumi, K.; Ooka, Y.; Kato, H.; Kanazawa, S.; Komatsu, S.; Nishi, E.; Hiroya, K.; Shigehisa, H. ChemRxiv DOI: 10.26434/chemrxiv.9981395.v1

(59) Zuckerman, D. S.; Woerpel, K. A. Diastereoselective peroxidation of derivatives of Baylis-Hillman adducts. Tetrahedron 2019, 75, 4118 .

( 60 ) Obradors, C.; Martinez, R. M.; Shenvi, R. A. $\mathrm{Ph}(i$-PrO $) \mathrm{SiH}_{2}$ : An Exceptional Reductant for Metal-Catalyzed Hydrogen Atom Transfers. J. Am. Chem. Soc. 2016, 138, 4962.

(61) Parsons, A. F.; Bar, G. Stereoselective radical reactions Chem. Soc. Rev. 2003, 32, 251; (b) Hanessian, S.; Yang, H, Schaum, R. Hydrogen-Bonding as a Stereocontrolling Element in Free-Radical C-Allylation Reactions: Vicinal, Proximal, and Remote Asymmetric Induction in the Amino Acid Series. J. Am. Chem. Soc. 1996, 118, 2507; (c) Pedrosa, R.; Andrés, C.; DuqueSoladana, J. P.; Mendiguchía, P. A Novel Case of Diastereoselection in 5-exo Radical Cyclization Promoted by Hydrogen Bonding Eur. J. Org. Chem. 2000, 3727; (d) Guindon, Y.; Guérin, B.; Landry, S.R., Intramolecular Aminyl and Iminyl Radical Additions to $\alpha, \beta$-Unsaturated Esters. Diastereoselective Tandem $\mathrm{Cy}$ clofunctionalization and Hydrogen Transfer Reactions. Org. Lett. 2001, 3, 2293.

(62) Heathcock, C.H.; Kath, J.C; Ruggeri, R. B. Daphniphyllum Alkaloids. 16. Total Synthesis of (+)-Codaphniphylline $J$. Org. Chem. 1995, 60, 1120.

(63) Xue, F.; Gu, W.; Silverman, R.B. Concise Route to the Chiral Pyrrolidine Core of Selective Inhibitors of Neuronal Nitric Oxide. Org. Lett. 2009, 11, 5194.

(64) González, D. F.; Brand, J. P.; Waser, J. Ethynyl-1,2benziodoxol-3(1H)-one (EBX): An Exceptional Reagent for the Ethynylation of Keto, Cyano, and Nitro Esters. Chem. Eur. J. 2010 s $16,9457$.

(65) Yudasaka, M.; Shimbo, D.; Maruyama, T.; Tada, N.; Itoh, A. Synthesis, Characterization, and Reactivity of an Ethynyl Benziodoxolone (EBX)-Acetonitrile Complex. Org. Lett. 2019, 21,1098 . 
(66) 3,5-dinitrobenzoate 33 was isolated and characterized, but it decomposed via facile benzoate hydrolysis during multiple crystallization attempts. The bis-(ethyl ester) corresponding to $\mathbf{3 3}$ was much more stable and easily crystallized (see Supporting Information).

(67) Bhagwat, S. S., Hamann, P. R., Still, W. C. Synthesis of Thromboxane A2.J. Am. Chem. Soc. 1985, 107, 6372.

(68) Bull, J. A.; Croft, R. A.; Davis, O. A.; Doran, R.; Morgan, K. F. Oxetanes: Recent Advances in Synthesis, Reactivity, and Medicinal Chemistry. Chem. Rev. 2016, 116, 12150.

(69) Wu, E., Kong, F., Synthesis and Conformational Analysis of Substituted 2,6-dioxabicyclo[3.3.1]heptanes: 1,3-anhydro2,4-di- $O$-benzyl- and -2,4-di- $O$-( $p$-bromobenzyl)- $\beta$-Lrhamnopyranose, Carbohydrate Res. 1987, 161, 235.

(70) Shimada, N.; Hasegawa, S.; Harada, T.; Tomisawa, T.; Fujii, A.; Takita, T. Oxetanocin, a Novel Nucleoside from Bacteria J. Antibiotics 1986, 39, 1623.

(71) Han, Q.; Zhang, J.; Lu, Y.; Wu, Y.; Zheng, Q.; Sun, H. A Novel Cytotoxic Oxetane ent-Kauranoid from Isodon japonicus. Planta Med. 2004, 70, 581.

(72) Emsermann, J.; Opatz, T.; Photochemical Approaches to the Bilobalide Core. Eur. J. Org. Chem. 2017, 3362.

(73) Maier, G.; Pfriem, S.; Schäfer, U.; Matusch, R. Tetratert-butyltetrahedrane. Angew. Chem. Int. Ed. Engl. 1978, 17, 520.

(74) Evans, D.A.; Kaldor, S.W.; Jones, T. K; Clardy, J.; Stout, T. J. Total synthesis of the macrolide antibiotic cytovaricin J. Am. Chem. Soc. 1990, 112, 7001 .

(75) Vedejs, E.; Chen, X. Parallel Kinetic Resolution. J. Am. Chem. Soc. 1997, 119, 2584.

(76) (a) Coric, I., Muller, S., List, B. Kinetic Resolution of Homoaldols via Catalytic Asymmetric Transacetalization. J. Am. Chem. Soc. 2010, 132, 17370; (b) Sun, Z.; Winschel, G. A.; Borokiva, A.; Nagorny, P. Chiral phosphoric acid-catalyzed enantioselective and diastereoselective spiroketalizations. J. Am. Chem. Soc. 2012, 134, 8074.

(77) We validated dr by NMR to avoid LC errors based on differential UV-activity, ionization of diastereomers, or impurity co-elution.

(78) (a) Shu, C.; Liu, M. Q.; Sun, Y. Z.; Ye, L. W. Efficient Synthesis of $\gamma$-Lactones via Gold-Catalyzed Tandem Cycloisomerization/Oxidation. Org. Lett. 2012, 14, 4958; (b) Zeng, M.; Herzon, S. B. Synthesis of 1,3-Amino Alcohols, 1,3-Diols, Amines, and Carboxylic Acids from Terminal Alkynes. J. Org. Chem. 2015, 80, 8604; (c) Asao, N.; Ohishi, T.; Sato, K.; Yamamoto, Y. Lewis acid catalyzed stereoselective hydrosilylation of ketones under the control of $\sigma-\pi$ chelation. Tetrahedron, 2002, 58, 8195 .

(79) Julia, M.; Saint-Jalmes, V. P.; Verpeaux, J.-N. Oxidation of Carbanions with Lithium Tert-Butyl Peroxide. Synlett 1993, 233 .

(80) Ohtawa, O.; Ogihara, S.; Sugiyama, K.; Shiomi, K.; Harigaya, Y.; Nagamitsu, T.; Ōmura, S. Enantioselective total synthesis of atpenin A5.J. Antibiot. 2009, 62, 289.

(81) Brown, H.C.; Bhat, N. G.; Srebnik, M. A Simple, General Synthesis of 1-Alkynyldiisopropoxyboranes. Tet. Lett. 1988, $29,2631$.

(82) This reaction was conceived and executed in October 2017 by Dr. Masaki Ohtawa, presented in November 2017 at ISONIS-11, posted to ChemRxiv (May 30, 2019) and published in Ref. 31 (October 16, 2019). A variation on this same reaction was published recently (March 27, 2020): Li, C.; Zhao, P.; Li, R.; Zhang, B.; Zhao, W. Oxidation of Alkynyl Boronates to Carbox- ylic Acids, Esters, and Amides. Angew. Chem. Int. Ed. 2020. DOI: $10.1002 /$ anie.202000988. A revision that cites our work is forthcoming.

(83) Nakanishi, K.; Habaguchi, K.; Nakadaira, Y.; Woods, M. C.; Maruyama, M.; Major, R. T.; Alauddin, M.; Patel, A. R.; Weinges, K.; Baehr, W. Structure of bilobalide, a rare tert-butyl containing sesquiterpenoid related to the C20-ginkgolides. J. Am. Chem. Soc. 1971, 93, 3544.

(84) (a) Lichtblau, D.; Berger, J. M.; Nakanishi, K. Efficient Extraction of Ginkgloides and Bilobalde from Ginkgo biloba Leaves J. Nat. Prod. 2002, 65, 1501-1504 (b) van Beek, T. A. Chemical analysis of Ginkgo biloba leaves and extracts J. Chromatogr. A. 2002, 967, 21-55. (c) Jaracz, S.; Malik, S.; Nakanishi, K. Phytochemistry 2004, 65, 2897-2902. (d) Choi, Y. H.; Kim, J.; Yoo, K.P Eupercritical-Fluid Extraction of Bilobalide and Ginkgolides from Ginkgo biloba Leaves by Use of a Mixture of Carbon Dioxide, Methanol, and Water Chromatographia 2002, 56, 753-757. (e) Lang, Q.; Wai, C. M., Pressurized water extraction (PWE) of terpene trilactones from Ginkgo biloba leaves Green Chemistry 2003, 5, 415-420.

(85) Van Beek, T.A., Comments on 'An Extraction Method for Determination of Ginkgolides ad Bilobalide in Ginkgo Leaf Extracts' Anal. Chem. 2000, 72, 3396.

(86) Fourtillan, J. B.; Brisson, A. M.; Girault, J.; Ingrand, I.; Decourt, J. P.; Drieu, K.; Jouenne, P.; Biber, A. Pharmacokinetic properties of Bilobalide and Ginkgolides A and B in healthy subjects after intravenous and oral administration of Ginkgo biloba extract (EGb 761). Thérapie 1995, 50, 137.

(87) Wang, J.; Ouyang, J.; Liu, Y.; Jia, X.; You, S.; He, S.; Di, X. Development of a sensitive LC-MS/MS method for the determination of bilobalide in rat plasma with special consideration of ex vivo bilobalide stability: Application to a preclinical pharmacokinetic study. J. Pharm. Biomed. Anal. 2014, 95, 238.

(88) Weinges, K.; Hepp, M.; Huber-Patz, U.; Rodewald, H.; Irngartinger, H. Chemie Der Ginkgolide, I. 10-Acetyl-1Methoxycarbonyl-2,3,14,15,16-Pentanorginkgolid A, Ein Zwischenprodukt Zur Herstellung von Bilobalid. Liebigs Ann. der Chemie 1986, 6, 1057.

(89) Lanthier, G. F.; Graham, W. A. G. $\pi$-Bonding in the Boron-Oxygen Bond. Chem. Comm. 1968, 715.

(90) Juaristi, E.; Cuevas, G. Recent Studies of the Anomeric Effect. Tetrahderon 1992, 48, 5019.

(91) Luger, P.; Kothe, Paulsen, H. Krisallstructur von 2,3,4tri- $O$-acetyl- $\beta$-D-xylopyranosylchloride. Carbohydr. Res. 1974, $37,283$.

(92) For example, acetylation of amides to imides lowers $\alpha$ CH acidity: Bordwell, F. G.; Fried, H. E. Heterocyclic Aromatic Anions with 4n $+2 \pi$-electrons. J. Org. Chem. 1991, 56, 4218 .

(93) Kochkodan, V.; Darwish, N .B.; Hilal, N. The chemistry of boron in water, in: Kabay, N.; Bryjak, M.; Hilal, N. (Eds.), Boron Separation Processes, Elsevier, Amsterdam, 2015.

(94) Chuang, K. V.; Xu, C.; Reisman, S. E. A 15-step synthesis of (+)-ryanodol. Science 2016, 353, 912.

(95) Related derivatives of $\mathbf{2}$ yielded similar pKa difference between inner/outer lactones (details in SI).

(96) For use in complexity-generation and biological annotation by 'cell-painting,' see: Gerry, C. J.; Hua, B, K.; Wawer, M. J.; Knowles, J. P.; Nelson Jr., S. D.; Verho, O.; Dandapani, S.; Wagner, B. K.; Clemons, P. A.; Booker-Milburn, K. I.; Boskovic, Z. V.; Schreiber, S. L. Real-Time Biological Annotation of Synthetic Compounds. J. Am. Chem. Soc. 2016, 138, 8920. 
(97) Li, J.; Eastgate, M. D. Current complexity: a tool for assessing the complexity of organic molecules, Org. Biomol. Chem. 2015, 13, 7164.

(98) Crossley, S. W. M.; Tong, G.; Lambrecht, M.; Burdge, H. E.; Shenvi, R. A. Synthesis of Picrotoxinin via Late-Stage Strong Bond Activations, DOI: 10.26434/ chemrxiv.8263415.v1

(99) Huffman, B.; Shenvi, R. A. Natural Products in the 'Marketplace': Interfacing Synthesis and Biology. J. Am. Chem. Soc. 2019, 141, 3332.

(100) Corey, E. J.; Wipke, W. T. Computer-assisted design of complex organic synthesis, Science 1969, 166, 178.

\section{TOC Graphic}

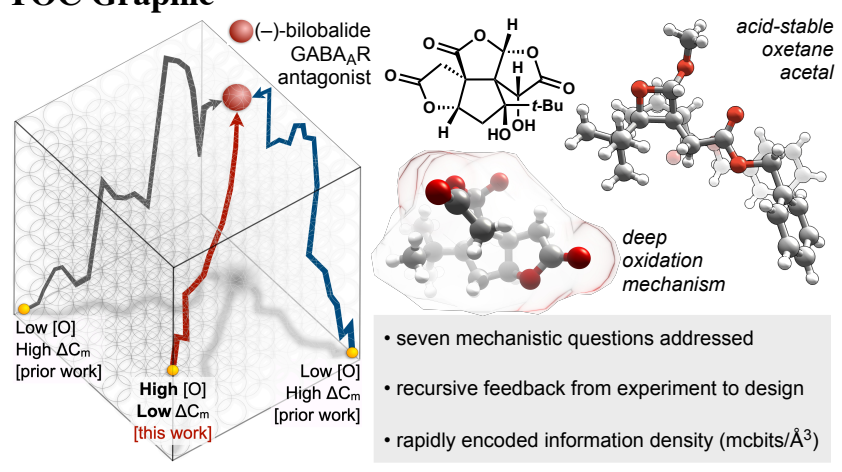

